\title{
Origin of Structural and Kinematic Properties of the Small Magellanic Cloud
}

\author{
Kenji Bekki $^{\mathrm{A}, \mathrm{C}}$ and Masashi Chiba ${ }^{\mathrm{B}}$ \\ A School of Physics, University of New South Wales, Sydney 2052, Australia \\ B Astronomical Institute, Tohoku University, Sendai, 980-8578, Japan \\ ${ }^{\mathrm{C}}$ Corresponding author. Email: bekki@phys.unsw.edu.au
}

Received 2008 August 29, accepted 2008 December 2

\begin{abstract}
We investigate structural, kinematic and chemical properties of stars and gas in the Small Magellanic Cloud (SMC) interacting with the Large Magellanic Cloud (LMC) and the Galaxy based on a series of selfconsistent chemodynamical simulations. We adopt a new 'dwarf spheroidal model' in which the SMC initially has both old stars with a spherical spatial distribution and an extended Hi gas disk. We mainly investigate the evolution of the SMC for the last 3 Gyr, during which the Magellanic Stream (MS) and the Magellanic Bridge (MB) could have formed as a result of the LMC-SMC-Galaxy interaction. Our principal results, which can be tested against observations, are as follows: The final spatial distribution of the old stars projected onto the sky is spherical, even after strong LMC-SMC-Galaxy interaction, whereas that of the new ones is significantly flattened and appears to form a bar structure. Old stars have a line-of-sight velocity dispersion $\sigma \simeq 30 \mathrm{~km} \mathrm{~s}^{-1}$ and slow rotation, with a maximum rotational velocity, $V<20 \mathrm{~km} \mathrm{~s}^{-1}$ and show asymmetry in the radial profiles. New stars have a smaller $\sigma$ than old ones and a significant amount of rotation $(V / \sigma>1)$. Hi gas shows velocity dispersions of $\sigma=10-40 \mathrm{~km} \mathrm{~s}^{-1}$, a high maximum rotational velocity $\left(V \sim 50 \mathrm{~km} \mathrm{~s}^{-1}\right)$ and a spatial distribution similar to that of new stars. New stars with ages younger than 3 Gyr show a negative metallicity gradient in the sense that more metal-rich stars are located in the inner regions of the SMC. The MB inevitably contains old stars with surface mass densities of $6-300 \times 10^{4} \mathrm{M}_{\odot} \mathrm{deg}^{-2}$ depending on initial stellar distributions of the modeled SMC. We find that the dwarf spheroidal model can explain more selfconsistently the observed kinematic properties of stars and gas, compared with another type of the model ('the disk model') in which the SMC initially consists of stellar and gas disks. We suggest that, to better understand its evolution, the SMC needs to be modeled as having a spheroidal component, rather than being a pure disk.
\end{abstract}

Keywords: galaxies: Magellanic Clouds — galaxies: star clusters — galaxies: stellar content — galaxies: interactions

\section{Introduction}

Dynamical interactions between the SMC, the LMC and the Galaxy have long been considered to be closely associated not only with the evolution of the SMC (e.g. Yoshizawa \& Noguchi 2003; YN03) but also with the formation of the MS (e.g. Putman et al. 1998) and the MB (e.g. Muller et al. 2004). The observed structural, kinematic and chemical properties of stars, star clusters and gas in the SMC have been extensively discussed in variously different aspects of the SMC, such as the star formation history (e.g. Russell \& Dopita 1992; Pagel \& Tautvaisiene 1998; Piatti et al. 2007), the total dynamical mass (e.g. Dopita et al. 1985; Stanimirović, StaveleySmith \& Jones 2004, hereafter S04) and the interaction history with the LMC (YN03; Bekki \& Chiba 2007). One of the key, longstanding problems related to the SMC as a galaxy is to understand how the past dynamical interaction between the SMC, the LMC and the galaxy could have influenced the chemical and dynamical evolution and the star-formation history of the SMC (e.g. Westerlund 1997).
In order to solve the above mentioned key problem, we need to know not only the three-dimensional (3D) space motions of the Magellanic Clouds (MCs) but also the dynamical and chemical properties of stars with different ages and metallicities. Recent proper motion measurements of the MCs and numerical studies of the orbits of the MCs based on the proper motion measurements have suggested that the MCs can be unbound to each other and that they are unbound to the Galaxy (Kallivayalil, van der Marel \& Alcock 2006, K06; Besla et al. 2007). The two-dimensional (2D) distributions of stellar populations with different ages and metallicities derived from AGB stars have revealed star formation histories in different local regions of the SMC (Cioni et al. 2006). Observational studies of stellar populations in the SMC based on their color-magnitude diagrams have revealed not only the $2 \mathrm{D}$ structures dependent on ages and metallicities, but also a more precise global star formation history of the SMC (e.g. Harris \& Zaritsky 2004). Thus, these observations of 2D structures of different stellar populations, if 
compared with the corresponding theoretical studies, can provide a vital clue to the above-mentioned key question of the SMC.

Previous theoretical models, however, did not provide useful predictions on the $2 \mathrm{D}$ (or 3D) structures of stars with different ages and metallicities, owing to strong limitations in their numerical simulations. YN03 discussed the star formation history of the SMC and the origin of structural and kinematic properties of the MS. They, however, did not discuss chemical and dynamical properties of stars and gas within the SMC. Although previous one-zone chemical evolution models discussed the long-term chemical evolution history of the SMC (e.g. Spite et al. 1988; Tsujimoto et al. 1995; Idiart, Maciel \& Costa 2007), they did not discuss dynamical properties of the SMC. Thus no theoretical studies have been done which attempt to investigate both dynamical and chemical properties of the SMC and thereby provide reasonable explanations for the abovementioned observations. Given that only chemodynamical simulations can provide predictions on 3D-dynamical and chemical properties in a self-consistent manner, it is vital for theoretical studies to investigate the above-mentioned key observational properties based on chemodynamical simulations of the SMC.

The purpose of this paper is thus to investigate numerically the physical properties of the SMC in a fully self-consistent manner and thereby try to provide answers for the above-mentioned unresolved problems. We mainly discuss the origin of structural, kinematic and chemical properties of the SMC's stellar and gaseous components derived from previous observations (e.g. Suntzeff et al. 1986; Torres \& Caranza 1987; Hardy, Suntzeff \& Azzopardi 1989; Russell \& Dopita 1992; Hatzidimitiriou et al. 1993; S04). We also discuss the latest observations that previous theoretical works have not discusssed: the structure of the stellar halo (Noël \& Gallart 2007), kinematic properties of older stellar populations (e.g. Harris \& Zarisky 2006), possibly purely gaseous streams in the MB (Harris 2007) and bifurcated structures and kinematics of the MC (Mathews \& Staveley-Smith, in preparation). However, in the present study, we do not intend to discuss some important aspects of the SMC evolution, such as the long-term evolution of the MCs and the formation of globular clusters (GCs), which were discussed to some extent in our previous papers (Bekki et al. 2004; Bekki \& Chiba 2005, hereafter paper I; Bekki 2007). We plan to discuss these in our paper III (Bekki \& Chiba, in preparation) based on more sophisticated and self-consistent models not only for the MCs but also for the Galaxy.

The layout of this paper is as follows. In Section 2, we summarize our numerical models used in the present study and describe the methods for analyzing structure and kinematics of the simulated SMC. In Section 3, we present numerical results on the time evolution of morphology, metallicity distribution and dynamical properties of the SMC. In Section 4, we discuss the above-mentioned outstanding issues related to formation and evolution of the SMC. The conclusions of the preset study are given in Section 5. Since the origin of the MS has been already discussed extensively in previous numerical simulations (e.g. Gardiner \& Noguchi 1996, hereafter GN96; Connors, Kawata \& Gibson 2006, C06), we briefly discuss it in the present paper. The present paper is still based on similar approaches used in previous numerical works (e.g. simple analytic models for dynamical friction and the fixed isothermal density profile for the Galactic dark-matter halo). More sophisticated models in which the LMC, the SMC and the Galaxy are represented by fully consistent $N$-body models will be described in our forthcoming paper (Bekki \& Chiba, in preparation).

\section{Model}

The present investigation is two-fold. First, we investigate the orbital evolution of the MCs with respect to the Galaxy for a given set of initial velocities of the MCs, by using a backward integration scheme (Murai \& Fujimoto 1980). Then we investigate chemodynamical evolution of the SMC on the orbits by using our original chemodynamical simulations with star formation models. Since the details of the methods for orbital calculations and numerical techniques for chemodynamical simulations of the MCs are given in the paper I, we briefly describe these in the present paper. The present model is significantly better than the previous ones (e.g. GN96 and C06) in the sense that (1) the SMC model is much more consistent with the observations and (2) star-formation and chemical-evolution processes are better modeled.

\subsection{Derivation of the Orbits of the MCs}

The gravitational potential of the Galaxy $\Phi_{\mathrm{G}}$ is assumed to have the logarithmic potential;

$$
\Phi_{\mathrm{G}}(r)=-V_{0}^{2} \ln r,
$$

where $r$ and $V_{0}$ are the distance from the Galactic center and the constant rotational velocity $\left(220 \mathrm{~km} \mathrm{~s}^{-1}\right)$, respectively. We consider this potential, firstly so the present model can be consistent with that of paper I (and those of previous papers, e.g. Murai \& Fujimoto 1980) and secondly because we can estimate the drag force due to dynamical friction between the MCs and the Galaxy in an analytical way for the orbital calculations of the MCs. Thanks to the adoption of this potential, we can compare the present results with previous ones (e.g. GN96; C06; K06; Besla et al. 2007).

Owing to the adopted isothermal density profile of the halo (i.e. ever increasing mass with radius), the MCs are more likely to show bound orbits for given initial velocities. If we adopt that the latest model for the Galaxy with a more realistic halo model (e.g. Widrow \& Dubinski 2005), the orbital evolution of the MCs can be different owing to the smaller mass of the Galaxy in its outer part $(R>100 \mathrm{kpc})$. However, it is not practical for the present study to adopt the model by Widrow \& Dubinski (2005), because analytical estimation of the drag force due to dynamical friction between the MCs and the Galaxy is not feasible. 
The LMC is assumed to obey the Plummer potential:

$$
\Phi_{\mathrm{L}}\left(r_{\mathrm{L}}\right)=\frac{-M_{\mathrm{LMC}}}{\sqrt{\left(r_{\mathrm{L}}^{2}+a_{\mathrm{L}}^{2}\right)}},
$$

where $M_{\mathrm{LMC}}, r_{\mathrm{L}}$ and $a_{\mathrm{L}}$ are the total mass of the LMC, the distance from the LMC and the effective radius, respectively. We adopt the same value of $a_{\mathrm{L}}=3 \mathrm{kpc}$ as previous numerical studies adopted (e.g. GN96). The SMC is also assumed to obey the Plummer potential:

$$
\Phi_{\mathrm{S}}\left(r_{\mathrm{S}}\right)=\frac{-G M_{\mathrm{SMC}}}{\sqrt{\left(r_{\mathrm{S}}^{2}+a_{\mathrm{S}}^{2}\right)}},
$$

where $M_{\mathrm{SMC}}, r_{\mathrm{S}}$ and $a_{\mathrm{S}}$ are the total mass of the SMC, the distance from the SMC and the effective radius, respectively. We adopt the same value of $a_{\mathrm{S}}=2 \mathrm{kpc}$ as previous numerical studies adopted (e.g. GN96).

We consider dynamical friction due to the presence of a Galactic dark-matter halo both for the LMC-Galaxy interaction and for the SMC-Galaxy interaction, and adopt the following expression (Binney \& Tremaine 1987):

$$
F_{\text {fric }, \mathrm{G}}=-0.428 \ln \Lambda_{\mathrm{G}} \frac{G M^{2}}{r^{2}}
$$

where $r$ is the distance of the LMC (the SMC) from the center of the Galaxy. The mass $M$ is either $M_{\mathrm{LMC}}$ or $M_{\mathrm{SMC}}$, depending on which Cloud's orbit (i.e. LMC or SMC) we calculate. We adopt the reasonable value of 3.0 for the Coulomb logarithm $\Lambda_{\mathrm{G}}$ (GSF; GN96) both in the orbital calculation of the LMC and in that of the SMC.

By integrating equations of the motions of the MCs toward the past from the present epoch, we investigate orbital evolution of the MCs for given $M_{\mathrm{LMC}}, M_{\mathrm{SMC}}$, initial positions and velocities of the MC. We adopt the reasonable sets of orbital parameters that are consistent with observations. The current Galactic coordinates $(b, l)$, where $l$ and $b$ are the Galactic longitude and latitude, respectively, are $(-32.89,280.46)$ for the LMC and $(-44.30,302.79)$ for the SMC. Therefore the current position $(X, Y, Z)$ of the $\mathrm{LMC},\left(X_{\mathrm{L}}, Y_{\mathrm{L}}, Z_{\mathrm{L}}\right)$ in units of $\mathrm{kpc}$, is $(-1.0,-40.8,-26.8)$ and that of the SMC, $\left(X_{\mathrm{S}}, Y_{\mathrm{S}}, Z_{\mathrm{S}}\right)$, is $(13.6,-34.3,-39.8)$. The current distance and the Galactocentric radial velocity of the LMC (SMC) is $80(7) \mathrm{km} \mathrm{s}^{-1}$.

The current space velocities or $(U, V, W)$ in units of $\mathrm{km} \mathrm{s}^{-1}$ are the most important parameters that determine the orbital evolution of the MCs in the present models. They are represented by $\left(U_{\mathrm{L}}, V_{\mathrm{L}}, W_{\mathrm{L}}\right)$ for the $\mathrm{LMC}$ and by $\left(U_{\mathrm{S}}, V_{\mathrm{S}}, W_{\mathrm{S}}\right)$ for the SMC. We mainly investigate the following two velocity types: The 'GN96 type' with $\left(U_{\mathrm{L}}, V_{\mathrm{L}}, W_{\mathrm{L}}\right)=(-5,-225,194)$ and $\left(U_{\mathrm{S}}, V_{\mathrm{S}}, W_{\mathrm{S}}\right)=(40,-185,171)$ and the 'K06 type' with $\left(U_{\mathrm{L}}, V_{\mathrm{L}}, W_{\mathrm{L}}\right)=(-86,-268,252)$ and $\left(U_{\mathrm{S}}, V_{\mathrm{S}}, W_{\mathrm{S}}\right)=$ $(-87,-247,149)$. The former is the same as those used in the models by GN96 whereas the latter is consistent with observations by K06.

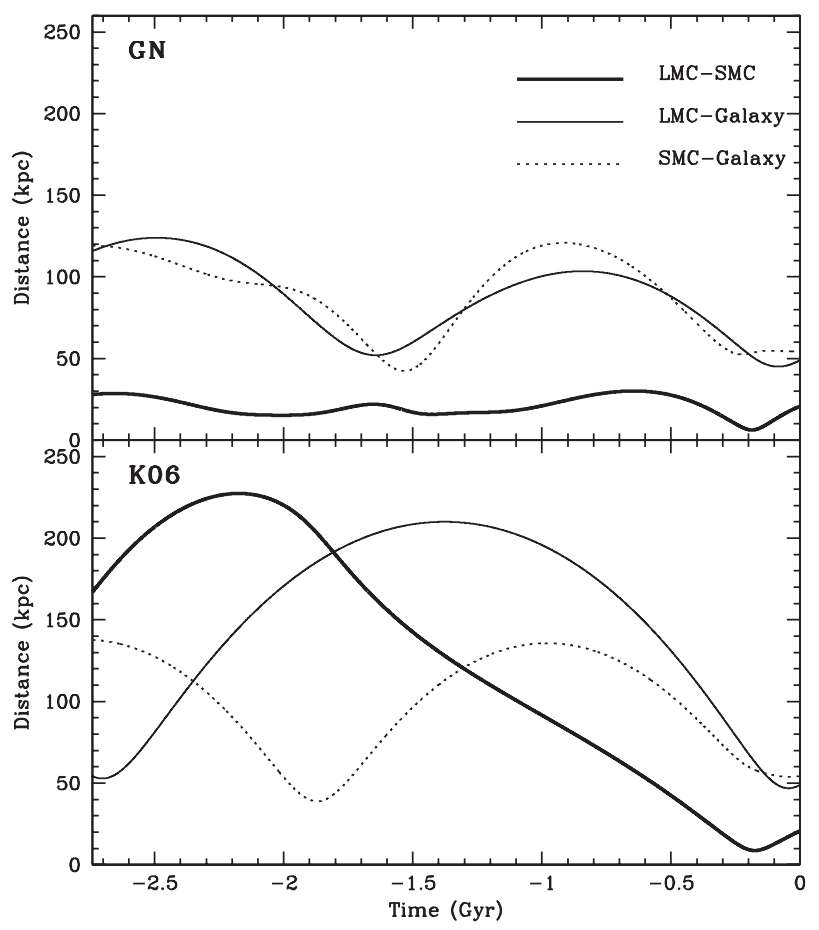

Figure 1 Orbital evolution of the MCs for the models $\mathrm{C} 1$ and C2 with the GN96 (upper) and the K06 (lower) velocity types. The time evolution of distances between MCs (thick solid), the LMC and the Galaxy (thin solid) and the SMC and the Galaxy (dotted) are separately shown. Here $M_{\mathrm{LMC}}=2.0 \times 10^{10} \mathrm{M}_{\odot}$ and $M_{\mathrm{SMC}}=3.0 \times 10^{9} \mathrm{M}_{\odot}$ are assumed for the two models.

Figure 1 describes the past $\sim 3-G y r$ orbital evolution of the MCs in the models with GN96 and K06 velocity types, for which $M_{\mathrm{LMC}}=2 \times 10^{10} \mathrm{M}_{\odot}$ and $M_{\mathrm{SMC}}=3 \times 10^{9} \mathrm{M}_{\odot}$ are assumed. Here negative values of the time, $T$, represent the past, with $T=0$ corresponding to the present epoch. As shown in this figure, the present orbital period of the MCs about the Galaxy is $\sim 1.5$ Gyr for the adopted gravitational potential and the masses of the MCs. The strong LMC-SMC interaction around $1.5 \mathrm{Gyr}$ can be seen only in the GN96-type model, which suggests that the MS can not be formed from the SMC in the K06-type model.

For a given set of initial positions and velocities of the MCs predicted by the above backward integration scheme, we dynamically evolve the MCs by using GRAPE-SPH simulations (i.e. integrating the equation of motion forward) for a fixed Galactic potential. Since the mass of the $\mathrm{SMC}$ is very small in comparison with the Galaxy, we consider that the tidal effects of the Galaxy on the SMC (e.g. stripping of stars from the SMC) can be properly treated by our models. Dynamical evolution of the SMC under the live potential of the Galaxy will be investigated by our future studies and the results will be compared with those of the present study.

We mainly discuss the models with 'classical' bound orbits of the LMC and the SMC (i.e. GN96-type) around the Galaxy in order to reproduce well the observed properties of the MS. The bound orbits are also suggested by Shattow \& Loeb (2008) who adopted $V_{0}$ a factor of 
$14 \%$ larger than $220 \mathrm{~km} \mathrm{~s}^{-1}$ adopted in the present study. They also suggested that the bound orbits can be consistent with the latest proper motion measurements by K05 as long as the higher circular velocity is adopted. Thus our main investigation of the GN96-type bound orbits can be justified in the present study.

\subsection{GRAPE-SPH Chemodynamical Simulations}

For a given orbit of the SMC, we investigate chemodynamical evolutions of the SMC by adopting the following two different galaxy models: (i) the 'dwarf spheroidal' model in which the SMC has a stellar spheroidal and an extended Hi gas disk and (ii) the 'disk' one in which the SMC has stellar and gaseous disks. Although the SMC is classified as an irregular dwarf of DDO type Ir IV-V (van den Bergh 2000), the usage of 'dwarf' spheroidal for the SMC would not be so appropriate, given that the SMC's stellar mass is as large as $10^{9} \mathrm{M}_{\odot}$ (S04): we use this term just for convenience.

Although the above disk model was adopted in previous models of the SMC (MK, GN96, YN and C06), we mainly investigate the dwarf spheroidal model in the present study. This is mainly because the dwarf spheroidal model can better explain the observed structural and kinematic properties of the SMC. The LMC is represented by a test particle with the mass of $M_{\mathrm{LMC}}$ and the Plummer potential described in Section 2.1 for most models. The results of simulations in which both the LMC and the SMC are represented by gaseous and stellar particles in a fully self-consistent manner will be described in (Bekki \& Chiba, in preparation) with main points of analysis significantly different from those of the present one.

\subsubsection{The Dwarf-Spheroidal Model}

A significant fraction of dwarf elliptical and spheroidal galaxies are observed to have exponential luminosity profiles (e.g. Ichikawa, Wakamatsu \& Okamura 1986; Andredakis \& Sanders 1994): we do not discriminate dwarf ellipticals and spheroids just for convenience in the present paper. Therefore the projected radial density profile $\left(\Sigma_{\mathrm{sph}}\right)$ of the stellar spheroid with the mass of $M_{\mathrm{sph}}$ and the scale length of $a_{\mathrm{sph}}$ is assumed to be an exponential one:

$$
\Sigma_{\mathrm{sph}}(R)=\Sigma_{\mathrm{sph}, 0} \times \exp \left(-R / a_{\mathrm{sph}}\right),
$$

where $\Sigma_{\mathrm{sph}, 0}$ and $R$ are the central surface density and the projected radius from the center of the spheroid (i.e. the center of the SMC), respectively. In order to derive the three dimensional (3D) density field $\left(\rho_{\mathrm{sph}}(r)\right.$, where $r$ is the distance from the center of the spheroid) from $\Sigma_{\mathrm{sph}}$, we can use the following formula (Binney \& Tremaine 1987):

$$
\rho_{\mathrm{sph}}(r)=-\frac{1}{\pi} \int_{r}^{\infty} \frac{d \Sigma_{\mathrm{sph}}(R)}{\mathrm{d} R} \frac{\mathrm{d} R}{\sqrt{R^{2}-r^{2}}} .
$$

We numerically estimate the $\rho_{\mathrm{sph}}(r)$ profile for a given $\Sigma_{\mathrm{sph}}(R)$ in a model with $M_{\mathrm{sph}}$ and $a_{\mathrm{sph}}$. The outer cut-off radius of the stellar spheroid $\left(R_{\mathrm{SMC}, \mathrm{s}}\right)$ is assumed to be a free parameter. The spheroid is assumed to be embedded in a massive dark-matter halo with the total mass of $M_{\mathrm{dm}}$ and the truncation radius of $R_{\mathrm{SMC}}$. This $R_{\mathrm{SMC}}$ represents the size of the SMC within which almost all particles are included. The radial density profile is assumed to have either the universal 'NFW' one with the central cusp (Navarro, Frenk \& White 1996) or a flat 'core' (Burkert 1995; Salucci \& Burkert 2000; referred to as the SB profile from now on). The NFW profile is described as:

$$
\rho_{\mathrm{dm}}(r)=\frac{\rho_{0}}{\left(r / r_{\mathrm{s}}\right)\left(1+r / r_{\mathrm{s}}\right)^{2}},
$$

where $r, \rho_{0}$ and $r_{\mathrm{s}}$ are the distance from the center of the cluster, the scale density and the scale-length of the dark halo, respectively. The SB profile is described as:

$$
\rho_{\mathrm{dm}}(r)=\frac{\rho_{\mathrm{dm}, 0}}{\left(r+a_{\mathrm{dm}}\right)\left(r^{2}+a_{\mathrm{dm}}^{2}\right)},
$$

where $\rho_{\mathrm{dm}, 0}$ and $a_{\mathrm{dm}}$ are the central dark matter density and the core (scale) radius, respectively. The halo is truncated at $7.5 \mathrm{kpc}$ from the center of the SMC.

S04 demonstrated that (i) the Hi rotation curve is very slowly rising within the central few kpc of the SMC with the maximum circular velocity of $\sim 60 \mathrm{~km} \mathrm{~s}^{-1}$ and (ii) the total mass of the SMC is $\sim 2.4 \times 10^{9} \mathrm{M}_{\odot}$ within the central $\sim 3 \mathrm{kpc}$. These observed properties of the SMC are better explained by the SB profile (for more detailed discussions on this see Bekki \& Stanimirović 2009). The NFW profile with reasonable parameters for the SMC (e.g. $c=5-20$, where $c$ is the ratio of the virial radius to $r_{\mathrm{s}}$ ) is not so consistent with the above observation (i). We find that the SB profiles with $a_{\mathrm{dm}}$ as large as $5-7 \mathrm{kpc}$ can be more consistent with the observations for $M_{\mathrm{SMC}}=3-4.5 \times 10^{9} \mathrm{M}_{\odot}$, though the slowly rising profile can not be exactly the same as the observed. We therefore show the results of the models with the SB profiles. We assume that $M_{\mathrm{dm}} / M_{\mathrm{sph}}$ is 2.33 and the halo is truncated at the tidal radius $\left(r_{\mathrm{t}}\right)$ of the SMC. The previous models for the SMC assumed that $r_{\mathrm{t}}$ ranges from $5 \mathrm{kpc}$ (GN96) to $7.5 \mathrm{kpc}(\mathrm{C} 06)$. $R_{\mathrm{SMC}}$ is the same as $r_{\mathrm{t}}$ in the present model and regarded as a free parameter.

The stellar spheroid is assumed to be supported purely by velocity dispersion and its dispersion is assumed to be isotropic. We therefore estimate the velocities of old stellar particles from the gravitational potential at the positions where they are located. In detail, we first calculate the one-dimensional isotropic dispersion according to the following formula:

$$
\sigma^{2}(r)=-\frac{U(r)}{3},
$$

where $U(r)$ is the gravitational potential at the position $r$ which is determined by the mass distributions of the dark-matter halo and the stellar spheroid. Then we allocate a velocity to stellar particle so that the distribution of velocities of these particles can have a Gaussian form with a dispersion equal to $\sigma^{2}(r)$. We adopt this method as 


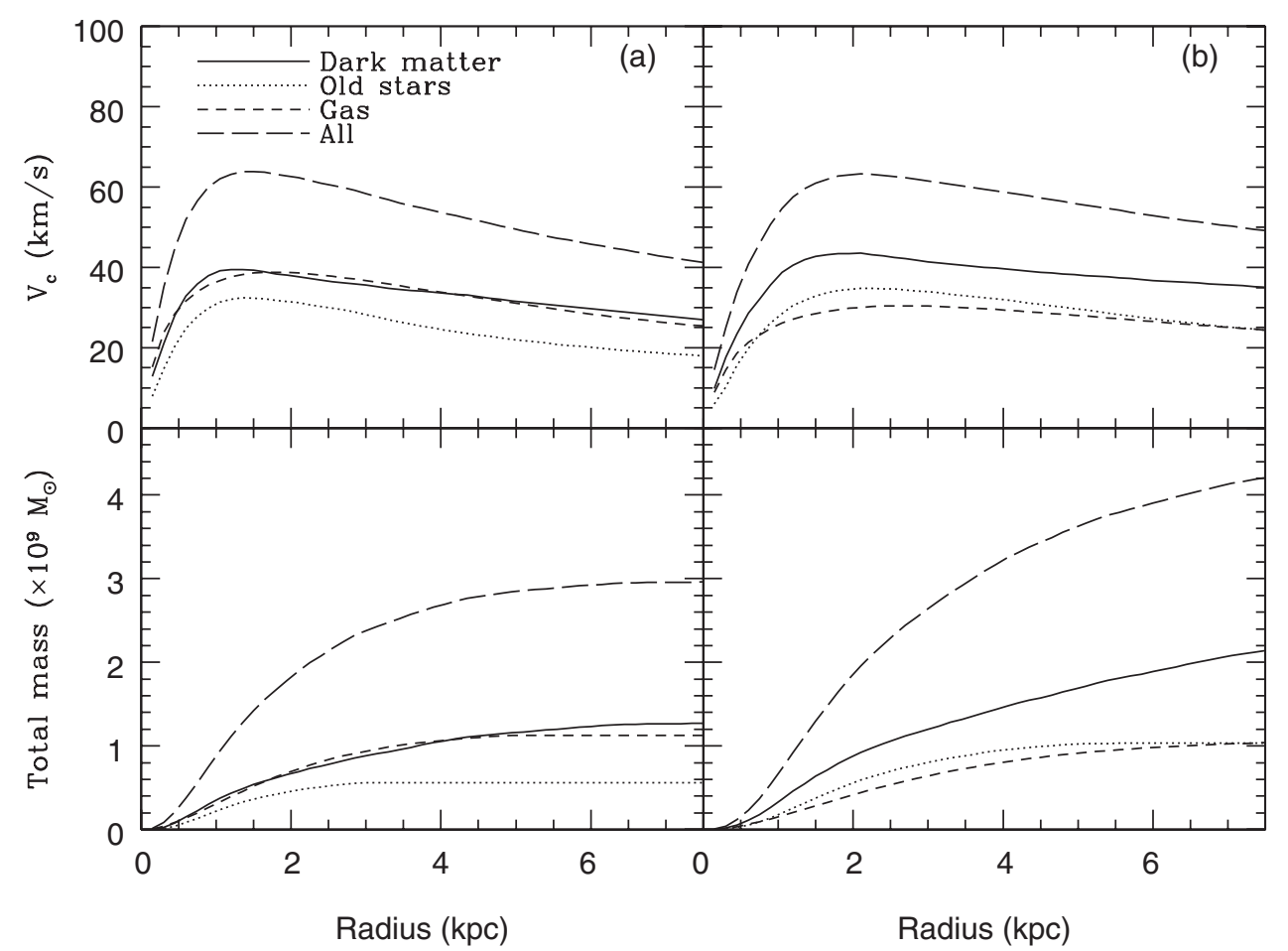

Figure 2 Circular velocities $\left(V_{\mathrm{c}}\right)$ dependent on the distance $(R)$ from the center of the SMC (upper) and total masses within $R$ (lower) for the model G5 with $M_{\mathrm{t}}=3.0 \times 10^{9} \mathrm{M}_{\odot}\left(\right.$ a) and G1 with $M_{\mathrm{t}}=4.5 \times 10^{9} \mathrm{M}_{\odot}(\mathrm{b}) . V_{\mathrm{c}}$ is calculated separately for the mass profiles of the dark-matter halo (solid), the old stars (dotted), the gas (short-dash) and all components (long-dash).

an approximation and thus need to let the halo dynamically relaxed to obtain a real dynamically equilibrium for a given distribution of the SMC: this approximation is quite useful, in particular, for implementing the triaxial potential of the disk model later described.

The SMC is assumed to have an extended Hi gas disk with the total gas mass of $M_{\mathrm{SMC}, \mathrm{g}}$, the size of $R_{\mathrm{SMC}, \mathrm{g}}$ and the exponential profile for the projected gaseous density. The scale length of the disk is set to be $0.2 R_{\mathrm{SMC}, \mathrm{g}}$ for all spheroidal models. The size ratio $\left(r_{\mathrm{g}}\right)$ of $R_{\mathrm{SMC}, \mathrm{g}}$ to $R_{\mathrm{SMC}, \mathrm{s}}$ is a key parameter which determines whether the MS is composed almost purely of gas (YN03). Furthermore Hi diameters of gas-rich galaxies are generally observed to be significantly larger than their optical disks (Broeils \& van Woerden 1994). Guided by these previous works, we investigate models with $2 \leq r_{\mathrm{g}} \leq 4$ in the present study. The mass ratio $\left(f_{\mathrm{g}}\right)$ of $M_{\mathrm{SMC}, \mathrm{g}}$ to $M_{\mathrm{SMC}, \mathrm{s}}$ is a key parameter which determines the masses of the MS and the LA (YN03). We find that $f_{\mathrm{g}}$ should be at least $\sim 2.0$ for $M_{\mathrm{SMC}}=3 \times 10^{9} \mathrm{M}_{\odot}$ and $\sim 1.0$ for $M_{\mathrm{SMC}}=4.5 \times 10^{9} \mathrm{M}_{\odot}$ to explain the total gas mass in the $\mathrm{MB}$ and the MS regions. We thus show the results of the models with $1 \leq f_{\mathrm{g}} \leq 2$ in the present study, though we have run models with $f_{\mathrm{g}} \leq 0.5$ as done in GN96 and C06.

Since the disk component in this spheroidal model is composed purely of gas, it is highly likely to be dynamically supported by rotation: gas dynamics (e.g. cooling and shock) in the disk can quickly dissipate away kinematic energy resulting from random motion of gas. Such a cold gas disk, however, can be quite unstable against dynamical instability (e.g. ring or bar instability), if the mass fraction of the disk is significant (e.g. Binney \& Tremaine 1987). For such a massive gas disk, the disk may well have a significant amount of kinetic energy due to random motion of the gas. In addition to the rotational velocity made by the gravitational field of the SMC, the initial radial and azimuthal velocity dispersion are thus given to the gaseous component according to the epicyclic theory with Toomre's parameter (Binney \& Tremaine 1987) $Q=1.5$. Thus the cold gas is stabilized by its random motion.

Since the SMC has a massive gaseous disk surrounding old stars, we need to adopt the following unique procedures to construct a dynamical equilibrium of the SMC. Firstly we give velocities of dark matter particles for given density profiles of the dark-matter halo and the stellar spheroid. Secondly we let the dark-matter halo evolve for ten dynamical time scales with the spheroid being fixed. During this relaxation process, the mass distribution of the halo can slightly change so that the system can reach a new dynamical equilibrium. Thirdly we allocate velocities for stellar particles according to Equation (9) and add a gas disk to the system. Fourthly, we again let the system evolve for ten dynamical time scales with the gas disk being fixed. During this relaxation process, the mass distribution of the spheroid (and the halo) can slightly change so that the system can reach a new dynamical equilibrium. Finally, we give rotational velocities to gaseous particles according to the circular velocities at their position for the new mass profile of the system.

Figure 2 shows the rotation curves of two dwarf spheroidal models with $M_{\mathrm{SMC}}=3.0 \times 10^{9} \mathrm{M}_{\odot} \quad$ and $R_{\mathrm{SMC}}=5.0 \mathrm{kpc}$ and with $M_{\mathrm{SMC}}=4.5 \times 10^{9} \mathrm{M}_{\odot}$ and 
$R_{\mathrm{SMC}}=7.5 \mathrm{kpc}$. These models show the maximum circular velocities of $\sim 60 \mathrm{~km} \mathrm{~s}^{-1}$, which is consistent with HI kinematics of the SMC (e.g. S04). For models with small SMC masses $\left(M_{\mathrm{SMC}} \sim 3.0 \times 10^{9} \mathrm{M}_{\odot}\right)$, the initial SMC size should be as small as $5 \mathrm{kpc}$ so that the final total mass of the SMC after efficient tidal stripping of stars and gas can be more consistent with the observations by S04. It should be stressed here that the rotation curves in this small-mass model reach their peaks at $\sim 1 \mathrm{kpc}$ and thus appear to be less consistent with the observations by $\mathrm{S} 04$. The total particle numbers for collisionless and collisional particles for the SMC are 100000 and 60000 , respectively. The total particle number of a MC system is 260000 (or larger, depending on whether the gas disk is modeled), when the LMC is also represented by a fully consistent disk model rather than a point-mass particle. The total mass of the dark-matter halo $\left(M_{\mathrm{dm}}\right)$ in a model with $M_{\mathrm{SMC}}=4.5 \times 10^{9} \mathrm{M}_{\odot}$ is about $2.4 \times 10^{9} \mathrm{M}_{\odot} ; M_{\mathrm{dm}}$ depends on $f_{\mathrm{g}}$ and $M_{\mathrm{SMC}}$.

\subsubsection{The Disk Model}

The radial $(R)$ and vertical $(Z)$ density profile of the initially thin disk of the SMC in this disk model are assumed to be proportional to $\exp \left(-R / R_{0}\right)$ with scale length $R_{0}=375 \mathrm{pc}$ and to $\operatorname{sech}^{2}\left(Z / Z_{0}\right)$ with scale length $Z_{0}=0.2 R_{0}$ respectively. The stellar disk is embedded in a massive dark-matter halo with the NFW radial density profile and the halo is truncated at the tidal radius of the $\operatorname{SMC}\left(r_{\mathrm{t}}=7.5 \mathrm{kpc}\right)$. The mass ratio of dark matter $\left(M_{\mathrm{dm}}\right)$ to the stellar disk $\left(M_{\mathrm{SMC}, \mathrm{s}}\right)$ is set to be 2.33 for all disk models. In addition to the rotational velocity made by the gravitational field of disk and halo component, the initial radial and azimuthal velocity dispersion are given to the disk component according to the epicyclic theory with Toomre's parameter (Binney \& Tremaine 1987) $Q=1.5$. The vertical velocity dispersion at a given radius is set to be 0.5 times as large as the radial velocity dispersion at that point.

The gaseous component in the disk model has the same properties as that in the spheroidal model. For consistency with the dwarf spheroidal models, we investigate the disk models with $1 \leq f_{\mathrm{g}} \leq 2$ and $2 \leq r_{\mathrm{g}} \leq 4$. Thus, the initial structures and kinematics of old stars are quite different in the dwarf spheroidal and disk models for the SMC whereas those of the gas are the same in the two models. These initial differences in stellar components can result in significant differences in dynamical properties and star formation histories of the SMC between the two different models. The particle numbers for the three components in the disk models are the same as those in the dwarf spheroidal model.

\subsubsection{Star Formation and Chemical Enrichment}

Star formation is modeled by converting the collisional gas particles into collisionless new stellar particles according to the algorithm of star formation described below. We adopt the Schmidt law (Schmidt 1959) with exponent $\gamma=1.5(1.0<\gamma<2.0$, Kennicutt 1998) as the controlling parameter of the rate of star formation. The amount of gas consumed by star formation for each gas particle in each time step is given as:

$$
\dot{\rho}_{\mathrm{g}}=C_{\mathrm{sf}} \rho_{\mathrm{g}}{ }^{\gamma} \text {, }
$$

where $\rho_{\mathrm{g}}$ and $C_{\mathrm{sf}}$ are, respectively, the gas density around each gas particle and a constant in the Schmidt law. The value of $C_{\text {sf }}$ is determined such that the star formation rate in the isolated model for the Galaxy with the disk mass of $6 \times 10^{10} \mathrm{M}_{\odot}$ and the gas mass fraction of 0.1 can be $\sim 1 \mathrm{M}_{\odot} \mathrm{yr}^{-1}$ (e.g. Bekki 1998). We convert a gas particle into a field star only if the local surface gas density exceeds the observed threshold gas density of $\sim 3 \mathrm{M}_{\odot} \mathrm{pc}^{-2}$ for Magellanic dwarf irregular galaxies (Hunter, Elmegreen \& Baker 1998). We estimate the surface gas density of a SPH particle from the volume gas density by assuming that the scale-height of the gas disk in the SMC being 100 pc during simulations. These stars formed from gas are called 'new stars' (or 'young stars') whereas stars initially within a disk are called 'old stars' throughout this paper.

Chemical enrichment from star formation and supernova feedback during the SMC evolution is assumed to proceed both locally and instantaneously in the present study. We assign the metallicity of the original gas particle to the new stellar particle and increase the metals of each neighbouring gas particle with the total number of neighbouring gas particles equal to $N_{\text {gas }}$, according to the following equation about the chemical enrichment:

$$
\Delta M_{\mathrm{Z}}=\frac{Z_{i} R_{\mathrm{met}} m_{\mathrm{s}}+\left(1.0-R_{\mathrm{met}}\right) \cdot\left(1.0-Z_{i}\right) m_{\mathrm{s}} y_{\mathrm{met}}}{N_{\mathrm{gas}}} .
$$

Here $\Delta M_{\mathrm{Z}}$ represents the increase of metal for each gas particle. $Z_{i}, R_{\text {met }}, m_{\mathrm{s}}$ and $y_{\text {met }}$ in the above equation represent the metallicity of the new stellar particle (or that of original gas particle) and the fraction of gas returned to interstellar medium, the mass of the new star and the chemical yield, respectively. The values of $R_{\text {met }}, y_{\text {met }}$ and the initial metallicity are set to be 0.3 and 0.004 and 0.0032 , respectively. For these values, the final mean metallicity of of the $\mathrm{SMC}$ is $[\mathrm{Fe} / \mathrm{H}]=-0.56$; this value is significantly smaller for a smaller $y_{\text {met }}$.

Dynamical evolution of the interstellar medium (ISM) can be significantly influenced by supernova explosions, and the effect of supernova explosions on the ISM depends on the initial mass function (IMF) adopted in the present study. For the stellar population formed in a GMC, we assume an IMF described by $\psi\left(m_{\mathrm{i}}\right)=A m_{\mathrm{i}}{ }^{-s}$, where $m_{\mathrm{i}}$ is the initial mass of each individual star and the slope of $s=2.35$ corresponds to the Salpeter IMF. The normalization factor $A$ is a function of $m_{\mathrm{cl}}$, which is a total mass of a stellar system, $m_{1}$ (lower mass cut-off) and $m_{\mathrm{u}}$ (upper one):

$$
A=\frac{m_{\mathrm{cl}} \times(2-s)}{m_{\mathrm{u}}^{2-s}-m_{1}^{2-s}} .
$$

$m_{\mathrm{u}}$ is a parameter and set to be $100 \mathrm{M}_{\odot}$ for most models whereas $m_{1}$ is regarded as a key free parameter in the 
present study. We shows the results of canonical models with $m_{1}=0.1 \mathrm{M}_{\odot}$ and $m_{\mathrm{u}}=100 \mathrm{M}_{\odot}$ and the top-heavy IMF ones with $m_{1}=1.0 \mathrm{M}_{\odot}$ and $m_{\mathrm{u}}=100 \mathrm{M}_{\odot}$. The mass fraction of stars that can explode as type-II supernova (i.e. $m_{\mathrm{i}}>8 \mathrm{M}_{\odot}$ ) is $14 \%$ for the canonical model and $35 \%$ for the top heavy IMF ones. About $10 \%$ of the total energy of one supernova $\left(\sim 10^{51} \mathrm{erg}\right)$ is assumed to be converted into kinematic energy of gas around the supernova (Thornton et al. 1998).

Some fraction of the kinematic supernova energy ( $E_{\mathrm{SN}, i}$ ) of the $i$ th stellar particle is assumed to be returned to gas particles (ISM) and then to change the kinetic energy of gas particles around the $i$ th stellar particle. Each $j$ th gas particle with the mass of $m_{g, j}$ around an $i$ th stellar particle can receive a velocity perturbation $\left(\Delta v_{\mathrm{SN}, j}\right)$ directed radially away from the $i$ th stellar particle. $\Delta v_{\mathrm{SN}, j}$ satisfies the relation

$$
f_{\mathrm{SN}} E_{\mathrm{SN}, i}=\frac{1}{2} \sum_{j=1}^{N_{\mathrm{nei}, i}} m_{g, j}\left(\Delta v_{\mathrm{SN}, j}\right)^{2},
$$

where $f_{\mathrm{SN}}$ represents the fraction of supernova energy returned to ISM, $N_{\text {nei, } i}$ is total number of gas particles that are located within the smoothing $\left(h_{\mathrm{i}}\right)$ radius around an $i$ th stellar particle. Although we investigate the models with $f_{\mathrm{SN}}=0$ (no feedback), $f_{\mathrm{SN}}=0.1$ and $f_{\mathrm{SN}}=1.0$ (maximum feedback), we show the results of the models with the more realistic $f_{\mathrm{SN}}=0.1$ (Thornton et al. 1998).

\subsubsection{Orbital Configurations}

The initial spin of a SMC (gas) disk in a model is specified by two angles, $\theta$ and $\phi$, where $\theta$ is the angle between the $Z$-axis and the vector of the angular momentum of a disk and $\phi$ is the azimuthal angle measured from $X$-axis to the projection of the angular momentum vector of a disk onto the $X-Y$ plane. Although we investigate many models with different $\theta$ and $\phi$, we mainly describe the results of the models with $\theta=45^{\circ}$ and $\phi=230^{\circ}$, This is mainly because these models can not only explain fundamental properties of the MS (GN96 and YN) but also reproduce dynamical properties of the present SMC.

\subsubsection{The GRAPE-SPH Simulations}

We use the GRAPE (GRAvity pyPE) system (e.g. Sugimoto et al. 1990) for the smoothed particle hydrodynamics (SPH) in order to evolve self-gravitating stellar and gaseous systems. Since we have already developed our original 'GRAPE-SPH' code capable of evolving selfgravitating systems composed both of collisionless and collisional masses (Bekki \& Chiba 2006), we use the code to simulate the evolution of the MCs for the last $\sim 3 \mathrm{Gyr}$. In this code, we calculate (1) mutual gravitational interactions between all particles and (2) the list of neighbor particles for each SPH gas particle by using the GRAPE. All other calculations related to hydrodynamics, star formation, chemical evolution and orbital evolution of the MCs are done by host computers for the GRAPE.
In order to implement SPH on the GRAPE system, we adopt numerical methods and techniques essentially the same as those used for the TREESPH method (e.g. Hernquist \& Katz 1989). We adopt the spherically symmetric spline kernel proposed by Monaghan \& Lattanzio (1985) and determine the smoothing lengths of SPH particles based on the numbers of their neighbor particles (Hernquist \& Katz 1989). We use the same forms of artificial viscosity as those used in Hernquist \& Katz (1989, Equations 2.22-2.25). We adopt an isothermal equation of state for the gas and determine the proper range of the initial gas temperatures $\left(T_{\mathrm{i}}\right)$ by running isolated disk models. We find that for the models with $T_{\mathrm{i}}=10^{4} \mathrm{~K}$, which is reasonable for luminous disk galaxies like the Galaxy, the initially thin gas disks quickly become very thick owing to the higher gaseous pressure. A smaller $T_{\mathrm{i}}$ is necessary for the SMC with its smaller mass and, thus, weaker selfgravity in the vertical direction. Although we investigate the models with $T_{\mathrm{i}}=250 \mathrm{~K}$ and $10^{3} \mathrm{~K}$, we mostly describe the results of models with $T_{\mathrm{i}}=250 \mathrm{~K}$.

We adopt the predictor-corrector algorithm that is accurate to 2nd order in time and space in order to integrate the equations describing the time evolution of self-gravitating systems in the present GRAPE-SPH code. The size of the time step $\left(\delta t_{\mathrm{i}}\right)$ for each SPH particle is determined by the Courant condition for the particle. $\delta t_{\mathrm{i}}$ for a SPH particle is derived from its gas density, sound velocity, velocity gradient and smoothing length, according to the formula adopted in Hernquist \& Katz (1989). The minimum and maximum possible sizes of time steps for SPH particles are set to be $1.67 \times 10^{2} \mathrm{yr}$ and $6.85 \times 10^{5} \mathrm{yr}$, respectively. We choose the above very small minimum possible size so that the time step size of every gas particle at a given time step can not be smaller than the minimum possible size: otherwise the simulation needs to stop. The size of the time step for a collisionless particle (dark matter, old and new stars) is fixed at $6.85 \times 10^{5} \mathrm{yr}$.

The gravitational softening lengths $(\epsilon)$ are different between collisionless and collisional particles and different between models with different initial SMC sizes $\left(R_{\mathrm{SMC}}\right)$. For the fiducial model (described later), $\epsilon=1.70 \mathrm{kpc}$ for collisionless particles and $\epsilon=199 \mathrm{pc}$ for collisional (gaseous) ones. Since our main interest is in structures on the scale of $1 \mathrm{kpc}$ and kinematics of the SMC, we consider that the resolution of the present simulation is high enough to investigate them.

\subsubsection{Stellar Population Synthesis Models}

We derive 2D distributions of the projected $B$-band luminosity densities $\left(\Sigma_{\mathrm{L}, \mathrm{B}}\right)$ of the SMC based on spatial distributions and age- and metallicity-distributions of old and new stars. In order to do this, we use the predictions of stellar population synthesis models by Vazdekis et al. (1996), which are available on his website. Since the observed metallicity of the stellar component of the SMC is with $[\mathrm{Fe} / \mathrm{H}]=-0.73 \pm 0.03$ (Luck et al. 1998), we use the $B$-band luminosities and stellar mass-to-light-ratios for the SSP models with $[\mathrm{Fe} / \mathrm{H}] \sim-0.68$ and variously 


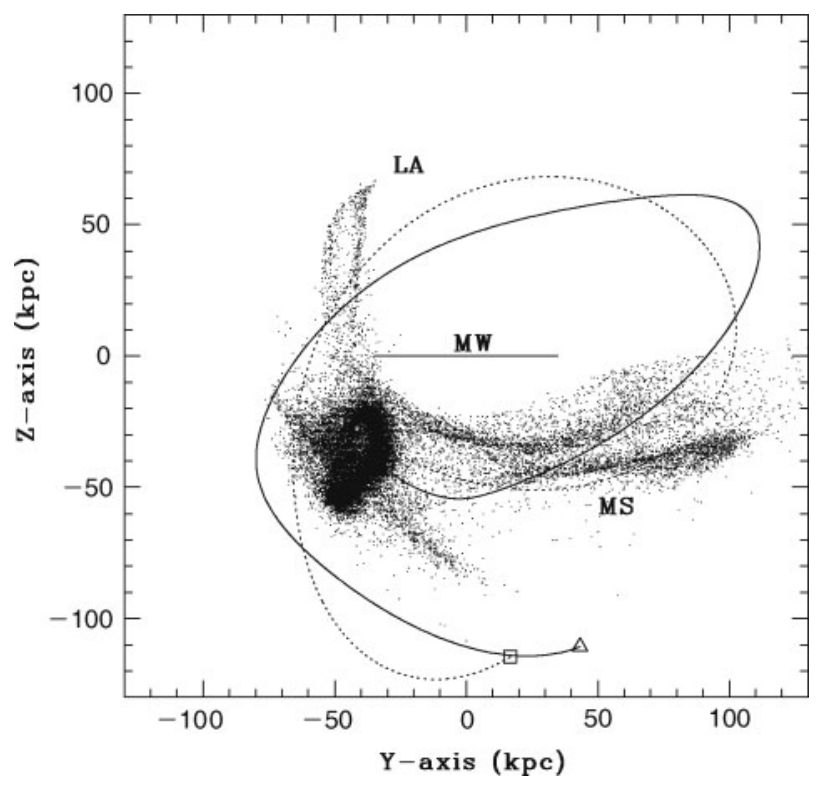

Figure 3 The final distribution of gas projected onto the $y-z$ plane for the model $\mathrm{C} 1$. The orbits of the LMC (triangle) and the SMC (square) with respect to the center of the Galaxy are shown by solid and dotted lines, respectively. For comparison, the Galaxy with the disk size of $17.5 \mathrm{kpc}$ is shown by a thick solid line.

different ages in Vazdekis et al. (1996). We assume that the mean ages of old and new stars in the present simulation are $5 \mathrm{Gyr}$ and $0.5 \mathrm{Gyr}$, respectively and thereby allocate the $B$-band luminosity to each stellar particle. We chose this set of stellar ages so that we can show how the SMC looks on the sky if the SMC has different distributions of old and new stars. Since our main focus in this paper is structural and kinematic properties of the SMC, we do not intend to discuss how the appearance of the SMC on the sky depends on ages of old and new stars.

We mainly investigate the projected $2 \mathrm{D} \Sigma_{\mathrm{L}, \mathrm{B}}$ distribution of the present SMC for the region with $0.1 \mathrm{~h}<\alpha<1.9 \mathrm{~h}$ and $-77^{\circ}<\delta<-69^{\circ}$ (corresponding roughly to the physical range of $4 \times 4 \mathrm{kpc}$ ), so that observations can be compared with the simulations. We divide the region into $50 \times 50$ cells and thereby estimate $\Sigma_{\mathrm{L}, \mathrm{B}}$ for each cell. We then smooth out the $2 \mathrm{D} \Sigma_{\mathrm{L}, \mathrm{B}}$ field by using the Gaussian kernel with the size 0.5 times the cell size so that we can clearly see the global $2 \mathrm{D} \Sigma_{\mathrm{L}, \mathrm{B}}$ distribution without unnecessarily dramatic cell-to-cell variations of $\Sigma_{\mathrm{L}, \mathrm{B}}$ due to small numbers of particles in some cells.

\subsection{Main Points of Analysis}

In the present GRAPE-SPH simulations, the SMC is modeled as a fully self-gravitating $N$-body system whereas the LMC is represented by a test particle with the abovementioned Plummer potential for most models. The mass distribution of the Galaxy is assumed to be fixed so that dynamical influences of the MCs on the Galaxy are not explicitly included in the models. We therefore do not intend to investigate dynamical and hydrodynamical interactions between the SMC, the LMC and the Galaxy: these interactions will be investigated in detail by Bekki \& Chiba (in preparation), in which the influences of the LMC-SMC interaction on the LMC's evolution and those of the LAGalaxy interaction on the evolution of the outer part of the Galactic Hi disk are discussed.

We consider that the best model of the SMC in the present study needs to reproduce reasonably well the projected locations of the MS and the LA in the Galactic coordinate $(l, b)$. Accordingly, we try to search for the models that can reproduce the observed locations of the MS and the LA among models with different model parameters. We first run 'collisionless models' (labeled as 'C') in which star formation and gas physics are switched off. If we find that a collisionless model reproduces the locations of the MS and the LA well, we then run a 'gas dynamical model' (labeled as 'G') with model parameters exactly the same as the collisionless model. Given that the present chemodynamical simulations are numerically costly, this two-fold investigation is quite effective at finding the best model of the SMC among G-type models. The LMC is represented by a fully self-consistent disk model (paper I) in some collisionless models so that we can confirm that the formation of the MS does not depend on whether the LMC is modeled as a test particle or a self-consistent disk composed of many particles.

Figure 3 shows the distribution of 'gas' particles with respect to the center of the Galaxy for the model $\mathrm{C} 1$, in which orbital evolution and masses of the MCs are consistent with previous models by GN96 and C06. This model can reproduce the observed location of the MS and the LA reasonably well and shows bifurcated structures in the MS, which appears to be consistent with observations. This model is therefore one of the successful models for the MS in the present study. The physical properties of the MS can be well reproduced by the present models with different $M_{\mathrm{SMC}}$, as long as we adopt the GN96 velocity type. However, models with the K06 velocity type cannot reproduce well the observed locations of the MS and the LAs, irrespective of $M_{\text {SMC }}$. The physical properties of the MS and the LAs in models with different velocity types are briefly discussed in Appendix A.

Although we ran 35 models with different model parameters, we describe here the results for 14 representative models that we regard as important in terms of the comparison with observations. The values of model parameters for 12 models are given in Table 1: the model numbers (column 1), $M_{\mathrm{SMC}}(2)$, velocity types (3), initial morphologies of the SMC (4), $R_{\mathrm{SMC}}(5), r_{\mathrm{s}}(6), f_{\mathrm{g}}$ (7), $r_{\mathrm{g}}(8), T_{\mathrm{i}}(9)$ and comments (10). We mainly describe the results of the 'fiducial model' in which the simulated properties of the SMC are more consistent with observations. Models with K06 velocity types fail to explain the observed locations of the MS and the LA in the galactic coordinate system. We however show and discuss the results of these in order to discuss the latest proper motion measurements of the MCs (K06; Piatek, Pryor \& Olszewski 2008) and orbital evolution models of the MCs based on them (Besla et al. 2007). 
Table 1. Model parameters for $N$-body simulations

\begin{tabular}{|c|c|c|c|c|c|c|c|c|c|}
\hline Model $^{\mathrm{a}}$ & $M_{\mathrm{SMC}}{ }^{\mathrm{b}}$ & Velocity types & Morphology & $R_{\mathrm{SMC}}{ }^{\mathrm{e}}$ & $r_{\mathrm{s}}^{\mathrm{f}}$ & $f_{\mathrm{g}} \mathrm{g}$ & $r_{\mathrm{g}}^{\mathrm{h}}$ & $T_{\mathrm{i}}^{\mathrm{i}}$ & Comments \\
\hline G1 & 0.45 & GN96 & dSph & 7.5 & 0.5 & 1.0 & 2.0 & 250 & Fiducial \\
\hline G2 & 0.45 & GN96 & $\mathrm{dSph}$ & 7.5 & 0.25 & 1.0 & 2.0 & 250 & More compact \\
\hline G3 & 0.45 & GN96 & disk & 7.5 & 0.25 & 1.0 & 4.0 & 250 & Disk model \\
\hline G4 & 0.45 & GN96 & dSph & 7.5 & 0.5 & 1.0 & 2.0 & 250 & $y_{\mathrm{met}}=0.002$ \\
\hline G5 & 0.30 & GN96 & $\mathrm{dSph}$ & 5.0 & 0.5 & 2.0 & 2.0 & 250 & Lower mass \\
\hline G6 & 0.30 & GN96 & $\mathrm{dSph}$ & 7.5 & 0.25 & 1.0 & 2.0 & 250 & \\
\hline G7 & 0.45 & K06 & $\mathrm{dSph}$ & 7.5 & 0.5 & 1.0 & 2.0 & 250 & Orbit from K06 \\
\hline I1 & 0.45 & - & dSph & 7.5 & 0.5 & 1.0 & 2.0 & 250 & Isolated \\
\hline I2 & 0.45 & - & $\mathrm{dSph}$ & 7.5 & 0.5 & 1.0 & 2.0 & $10^{3}$ & \\
\hline $\mathrm{I} 3$ & 0.45 & - & $\mathrm{dSph}$ & 7.5 & 0.5 & 1.0 & 2.0 & $10^{4}$ & \\
\hline $\mathrm{C} 1$ & 0.30 & GN96 & $\mathrm{dSph}$ & 7.5 & 0.25 & 2.0 & 2.0 & 250 & Collisionless \\
\hline $\mathrm{C} 2$ & 0.30 & K06 & $\mathrm{dSph}$ & 7.5 & 0.25 & 2.0 & 2.0 & 250 & \\
\hline $\mathrm{C} 3$ & 0.45 & GN96 & $\mathrm{dSph}$ & 7.5 & 0.5 & 1.0 & 2.0 & 250 & \\
\hline C4 & 0.30 & GN96 & $\mathrm{dSph}$ & 5.0 & 0.5 & 2.0 & 2.0 & 250 & \\
\hline
\end{tabular}

a ' $G$ ' represents gaseous models in which gas physics, star formation in gas and chemical enrichment processes are all included, whereas ' $\mathrm{C}$ ' represents collisionless models in which such processes are switched off. 'I' represents isolated models in which interaction between the MCs and the Galaxy is not included at all.

${ }^{\mathrm{b}} \mathrm{The}$ total mass of the SMC in units of $10^{10} \mathrm{M}_{\odot}$.

c 'GN96' and 'K06' describe the orbits of the MCs that are consistent with those by GN96 and K06, respectively.

dThe initial morphology of old stars: 'dSph' and 'disk' represent the dwarf spheroidal and the disk models, respectively. We use the term of dwarf spheroid for convenience, though it would not be so appropriate for the SMC to be classified as a 'dwarf' spheroid for its luminosity.

${ }^{\mathrm{e}} \mathrm{The}$ size of the SMC which corresponds to the tidal radius in units of kpc.

${ }^{\mathrm{f}}$ The ratio of the stellar disk size $\left(R_{\mathrm{SMC}, \mathrm{s}}\right)$ to the SMC one $\left(R_{\mathrm{SMC}}\right)$.

gThe mass ratio of gas to stars in the SMC.

${ }^{\mathrm{h}}$ The size ratio of the gaseous disk to the stellar spheroid or disk in the SMC.

${ }^{\mathrm{i}}$ The initial gaseous temperate in units of $\mathrm{K}$. The low temperature of cold gas is adopted so that the outer disk can keep its initial thin configuration for most models.

We also investigate the 'isolated model' with no LMCSMC-Galaxy interaction in order to confirm that the derived characteristic properties of the SMC in the G models are due to the LMC-SMC-Galaxy interaction. We investigate three isolated models (I1, I2 and I3) with different $T_{\mathrm{i}}$ so that we can confirm which model(s) can be dynamically stable. We find that the gas disks in models with higher $T_{\mathrm{i}}$ (e.g. I3 with $T_{\mathrm{i}}=10^{4} \mathrm{~K}$ ) become unrealistically thick owing to high gaseous pressure not reasonable for the SMC with a small mass. We also find that the models with $T_{\mathrm{i}}=250 \mathrm{~K}$ can keep initially thin gas disks and continue to be stable. We therefore show the results of the $\mathrm{G}$ models with $T_{\mathrm{i}}=250 \mathrm{~K}$ in the present study.

\section{Results}

\subsection{Fiducial Model}

\subsubsection{Dynamical Evolution}

Figure 4 shows how the total masses of dark matter, old stars, new ones and gas within the central $5 \mathrm{kpc}$ of the SMC evolve with time during strong tidal interaction between the SMC, the LMC and the Galaxy in the fiducial model G1. The dark mater, old stellar and gaseous components lose $40 \%, 41 \%, 75 \%$ of their initial masses during the tidal interaction. The larger fraction of gas mass lost during the interaction is due both to more efficient tidal stripping of gas and to gas consumption by star formation. The central spheroidal component (i.e. old stars) in this model can not be so strongly influenced by the tidal fields owing to its initial compactness so that it does not lose its mass during the strong LMC-SMC interaction around $T=-1.5 \mathrm{Gyr}$. It however loses a significant fraction of old stars at the last LMC-SMC interaction about 0.2 Gyr ago so that the MB composed of old stars can be formed. The final total mass within the central $5 \mathrm{kpc}$ in this model is $2.4 \times 10^{9} \mathrm{M}_{\odot}$, which is consistent with the observed total mass of the SMC (e.g. S04).

Figure 4 also shows that not only stars but also gas initially in the SMC can pass through the central $7.5 \mathrm{kpc}$ of the LMC (corresponding to the disk size), in particular, at $T=-1.4$ and $-0.2 \mathrm{Gyr}$. This result confirms the 'Magellanic squall' (Bekki \& Chiba 2007), which means efficient mass-transfer from the SMC to the LMC via the LMC-SMC tidal interaction. Figure 4 furthermore shows that (i) new stars formed in the SMC can pass through the LMC and (ii) the total mass of the SMC's new stars that are located within the LMC at $T=0$ is about $4 \times 10^{7} \mathrm{M}_{\odot}$. These results imply that metal-poor $([\mathrm{Fe} / \mathrm{H}]<-0.6)$, younger stars (or star clusters) with ages less than $3 \mathrm{Gyr}$ currently observed in the halo region of the LMC might well originate from the SMC. We note that Muñoz et al. (2006) recently discovered metal-poor stars with possible metallicities of $[\mathrm{Fe} / \mathrm{H}]=-0.67$ in the outer halo of the LMC, though they did not estimate ages of these stars. Owing to the total mass of the LMC being 


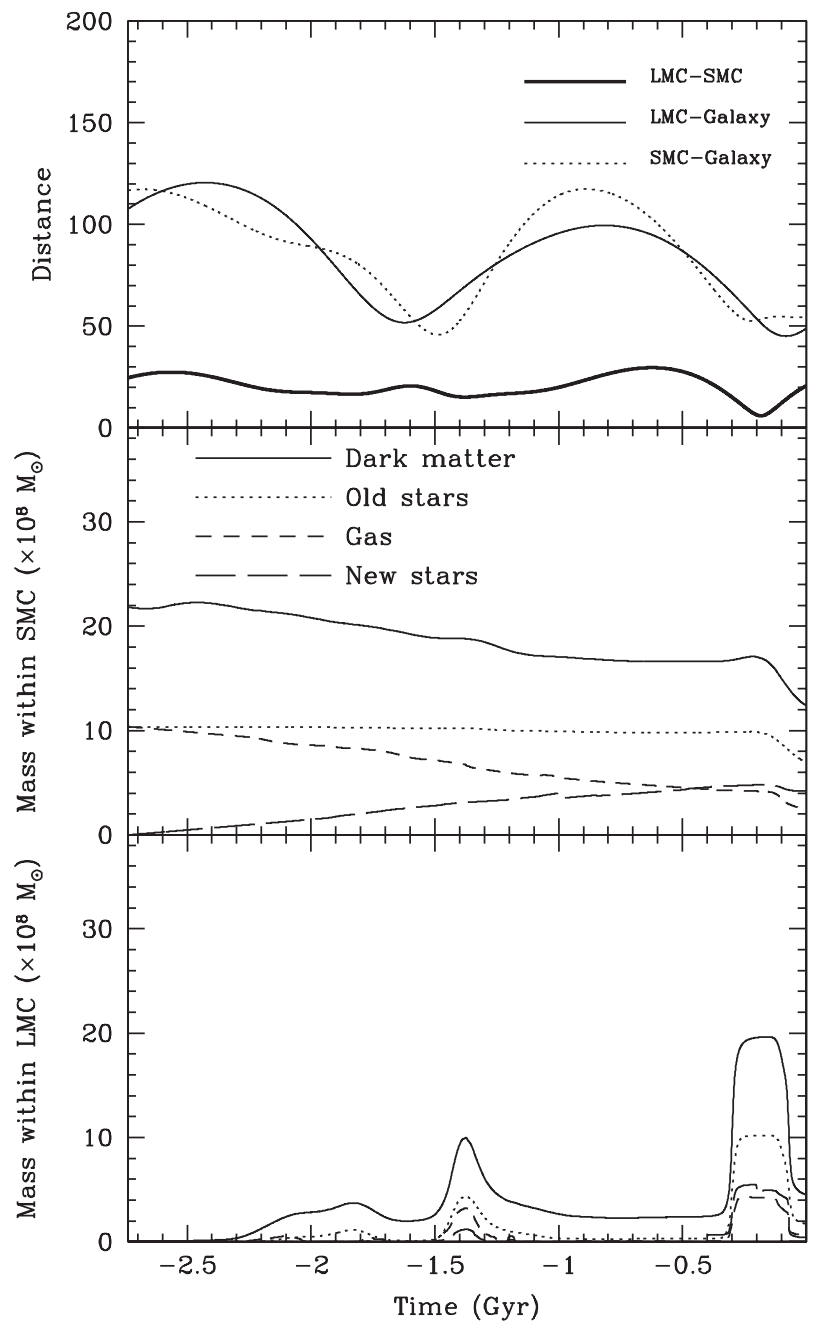

Figure 4 The top panel shows the time evolution of the distances between the LMC-SMC (thick solid), the LMC-Galaxy (thin solid) and the SMC-Galaxy (dotted) for the fiducial model G1. The middle panel shows the time evolution of the total masses within the central $5 \mathrm{kpc}$ of the SMC for the dark matter (solid), old stars (dotted), gas (short-dash) and new stars (long-dash). The bottom panel shows the time evolution of the total masses the dark matter (solid), old stars (dotted), gas (short-dash) and new stars (long-dash) that are initially in the SMC and later in the central $7.5 \mathrm{kpc}$ of the $\mathrm{LMC}$ at a give time step.

significantly larger than that of the SMC, mass transfer from the LMC to the SMC does not occur.

The strong tidal interaction can also strongly compress the gas to form high-density asymmetric structures (e.g. spirals), where star formation is moderately enhanced (up to $\sim 0.4 \mathrm{M}_{\odot} \mathrm{yr}^{-1}$ ). Owing to the absence of the stellar bar in the dwarf spheroid, a nuclear starburst is not induced by the interaction: this result is in a striking contrast with those by Noguchi (1988) in which stellar bars formed in the disks of interacting galaxies can trigger nuclear starbursts. About $30 \%$ of the initial gas is tidally stripped to form the MS, the LAs and the MB, depending on the present locations of the gas. The stellar spheroid can not be strongly distorted by the tidal interaction so that the final morphology of the SMC shows an old stellar spheroid with a gaseous disk more compact than the initial one and

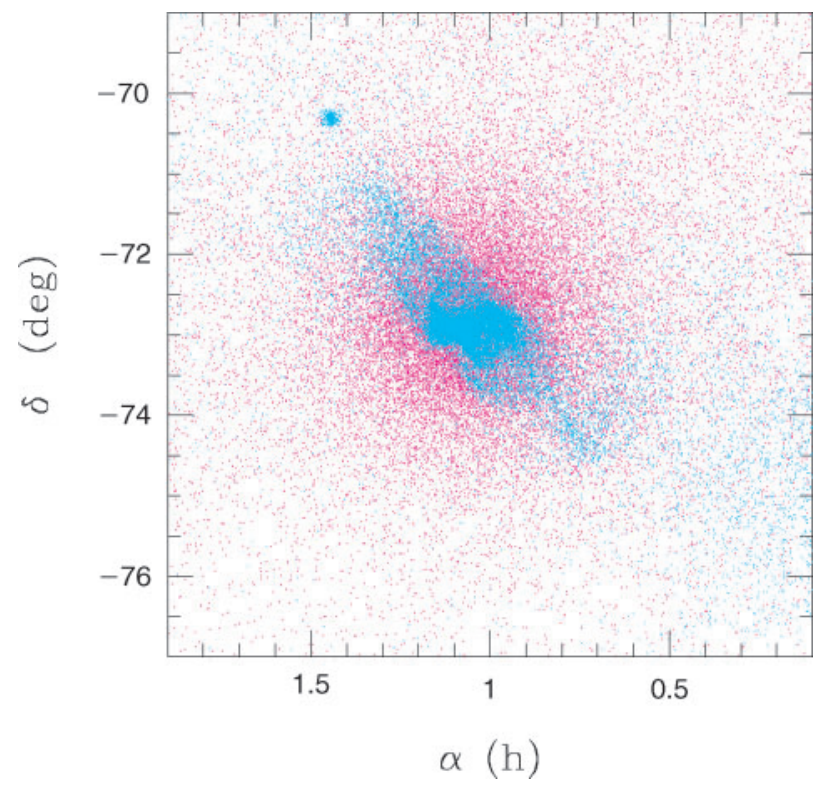

Figure 5 The final distributions of old stars (magenta) and new ones (cyan) on the sky (i.e. in the $\alpha-\delta$ coordinate system) for the fiducial model (G1).
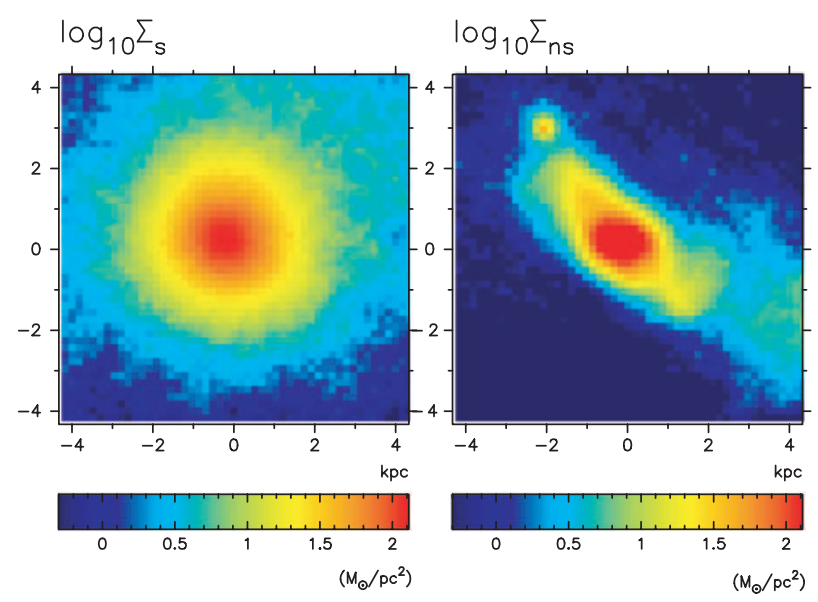

Figure 6 The final 2D distributions of projected mass densities $\left(\Sigma\right.$ in units of $\left.\mathrm{M}_{\odot} \mathrm{pc}^{-2}\right)$ for old stars $\left(\Sigma_{\mathrm{s}}\right)$ and new ones $\left(\Sigma_{\mathrm{ns}}\right)$ in logarithmic scales for the fiducial model. Note that the flattened structure can be clearly seen in this simulated distributions.

a compact stellar one composed only of young stars with ages less than $2.7 \mathrm{Gyr}$.

\subsubsection{Stellar Structures and Kinematics}

As shown in Figure 5, the final projected spatial distribution on the sky (i.e. on the $\alpha-\delta$ coordinate system) are quite different between old and new stars. New stars originate from the gas disk disturbed and compressed by the tidal fields of the LMC and the Galaxy so that the final distribution of new stars is quite elongated: this structure is reminiscent of the observed apparently 'barred structure' in the SMC. The old stars, on the other hand, are not so strongly influenced by the tidal fields as the gas owing to their initial compact distribution so that they can not be so tidally elongated at $T=0$. They appear to show an almost spherical shape on the sky, though the spherical 


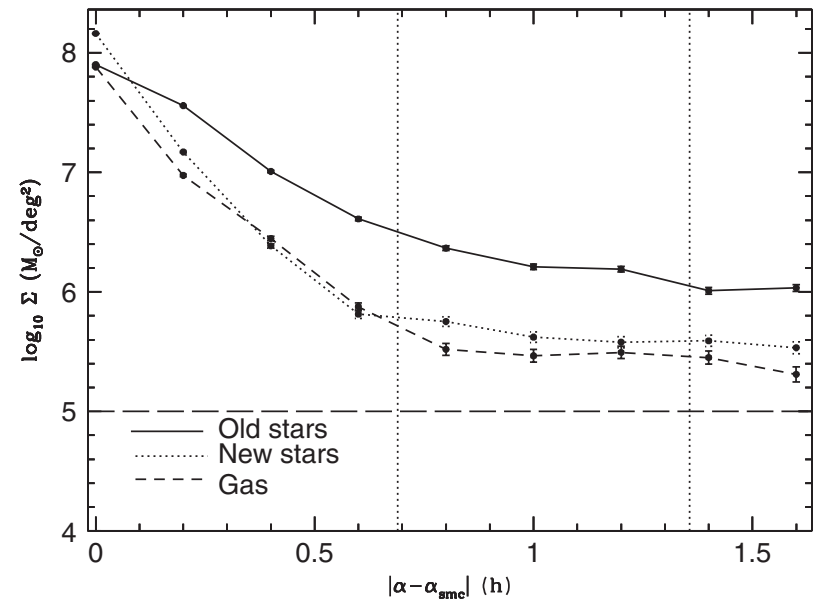

Figure 7 The final projected mass density profiles $(\Sigma)$ in units of $\mathrm{M}_{\odot} \operatorname{deg}^{-2}$ along $\alpha$ for old stars (solid), new ones (dotted) and gas (dashed) in the fiducial model. The profiles are derived for for particles with $\alpha>\alpha_{\mathrm{SMC}}$, where $\alpha_{\mathrm{SMC}}$ is $\alpha$ of the SMC, so that the mass profiles in the MB regions can be clearly seen. For comparison, the MB region where HI and molecular observations (e.g. Mizuno et al. 2006) have been done is shown by two dotted lines.

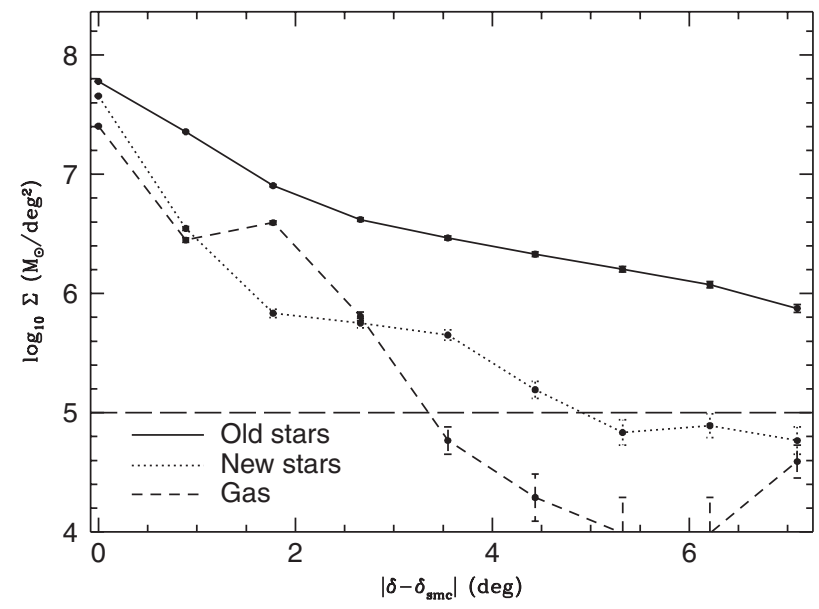

Figure 8 The same as Figure 7 but along $\delta$. The profiles are derived for for particles with $\delta>\delta_{\mathrm{SMC}}$, where $\delta_{\mathrm{SMC}}$ is $\delta$ of the SMC, so that the observed mass profiles of the stellar halo can be compared with the simulation. The physical ranges of $\alpha$ and $\delta$ in Figures 7 and 8 are the same so that the profiles along $\alpha$ and $\delta$ can be compared with each other.

morphology is not so clearly seen in this plot because of the stripped stars being widely distributed in the outer halo region of the SMC. These results clearly explain why there are significant differences between the morphology of old stellar populations (e.g. red giant stars, Harris \& Zarisky 2006) and the optical morphology depending strongly on young stellar populations. A GC-like compact stellar system that is formed during the tidal interaction and thus composed of new stars can be seen in the upper left corner of the plot, i.e. $(\alpha, \delta) \approx\left(1.5 \mathrm{~h},-70.5^{\circ}\right)$.

Figure 6 shows that the 2D distributions of the projected surface mass densities $(\Sigma)$ for old stars $\left(\Sigma_{\mathrm{s}}\right)$ and for new ones $\left(\Sigma_{\mathrm{ns}}\right)$ that are produced based on the projected distributions of stars for the region shown in Figure 5.

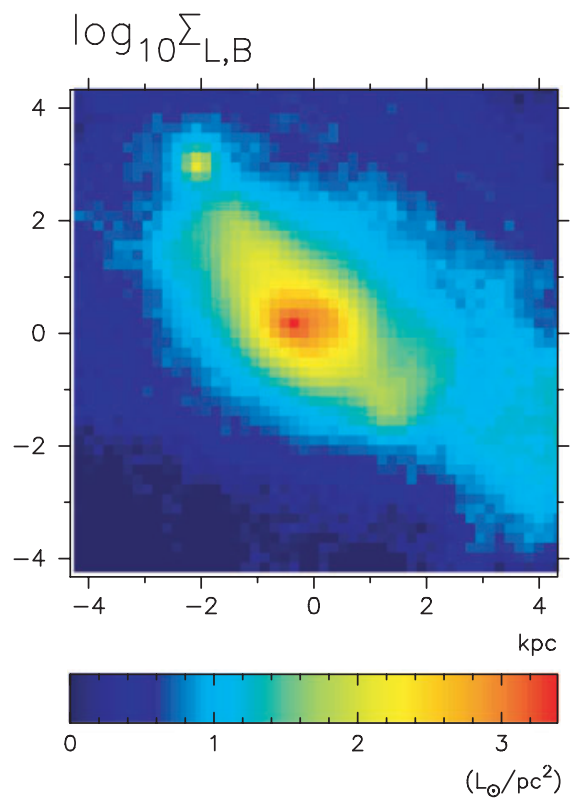

Figure 9 The final projected 2D distribution of the $B$-band luminosity density $\left(\Sigma_{\mathrm{L}, \mathrm{B}}\right)$ of the SMC for the fiducial model. The distribution is produced based on the mass distributions shown in Figure 6 for adopted stellar population models. The details of the methods to produce the distribution are given in the main text.

The old stellar component clearly shows a spherical distribution on the sky, though $\Sigma_{\mathrm{s}}$ of the outer halo is slightly enhanced in the northern direction. This northsouth asymmetry in the outer stellar halo is highly likely to be due to the past SMC-LMC-Galaxy tidal interaction. The new stellar component is significantly elongated and the major axis of the distribution in the central region $(R<1 \mathrm{kpc})$ is not coincident with that of the outer part $(R>2 \mathrm{kpc})$. The lower part appears to be more strongly disturbed than the upper one owing to the last LMC-SMC tidal interaction about $0.2 \mathrm{Gyr}$ ago.

Owing to the efficient tidal stripping of old stars in the last LMC-SMC interaction about $0.2 \mathrm{Gyr}$ ago, the SMC loses a significant fraction $(\sim 40 \%)$ of old stars. As a result of this, the stripped stars can be widely distributed between the LMC and the SMC (i.e. the MB region). Figure 7 shows that the projected surface mass density $(\Sigma)$ of old stars in the MB region has a relatively flat distribution with $\Sigma$ being significantly higher than $10^{6} \mathrm{M}_{\odot} \mathrm{deg}^{-2}$. The presence of this extended halo-like component is due largely to the stripped. The surface density of new stars in the MB region is significantly lower than that of old ones in this model, though the $\Sigma$-ratio of old to new stars in the MB depends on the initial distributions of old stars in the SMC, as discussed later in this paper. As shown in Figure 7 , there are no significant differences in the $\Sigma$ profiles between gas and new stars in the MB.

Figure 8 shows that the $\Sigma$ profiles of old and new stars and gas along $\delta$ appear to be significantly different from those along $\alpha$, which suggests that the global mass distribution in the outer part of the SMC is highly asymmetric owing to the past interaction with the LMC and 


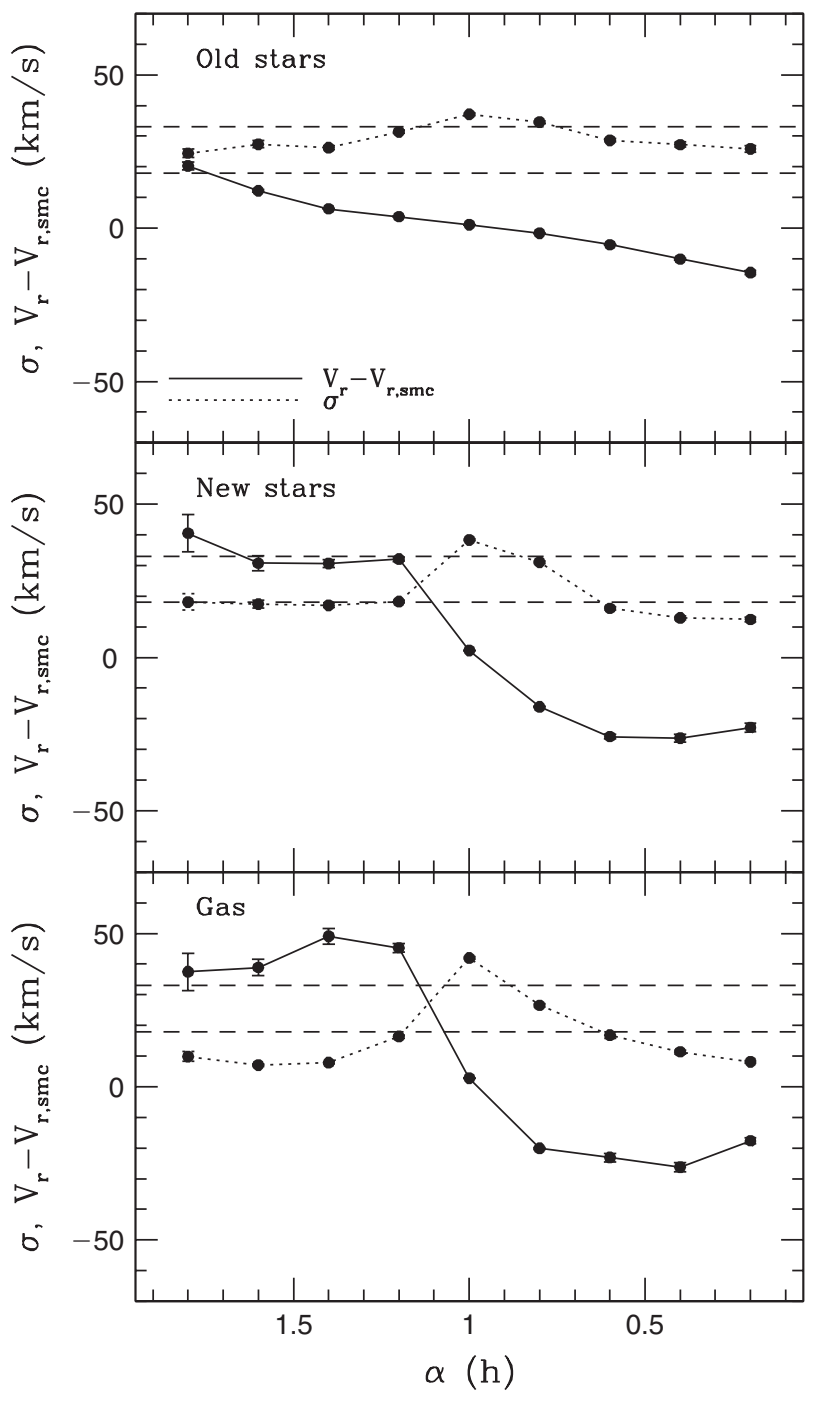

Figure 10 The final profiles of line-of-sight velocities $\left(V_{\mathrm{r}}-V_{\mathrm{r}, \mathrm{SMC}}\right.$, solid) and dispersions ( $\sigma$, dotted) for old stars (top), new ones (middle) and gas (bottom) in the fiducial model, where $V_{\mathrm{r}}$ and $V_{\mathrm{r}, \mathrm{SMC}}$ are the line-of-sight velocity of particles and of the center of the SMC, respectively. For comparison, the observed range of the velocity dispersions of older stars is shown by dashed lines.

the Galaxy. $\Sigma$ of gas and new stars along $\alpha$ are systematically higher that those along $\delta$ for the outer halo region $\left(\left|\alpha-\alpha_{\mathrm{SMC}}\right|>0.7 \mathrm{~h}\right.$ or $\left.\left|\delta-\delta_{\mathrm{SMC}}\right|>3^{\circ}\right)$ which means that a larger fraction of the stripped stars and gas are located in the MB region. There are no remarkable differences in $\Sigma$ profiles along $\alpha$ and $\delta$ for old stars in the SMC. These results on the possible differences in $\Sigma$ profiles along $\alpha$ and $\delta$ between stellar and gaseous components will be discussed later in the context of the formation of the MB and the outer stellar halo in the SMC.

Figure 9 shows that the 2D $B$-band surface brightness $\left(\Sigma_{\mathrm{L}, \mathrm{B}}\right)$ distribution of the SMC has an irregular shape rather than a regular spherical one, which is expected from the dominant old stellar populations in this model. This result clearly suggests that the observed irregular appearance in the SMC is due largely to the distribution of young

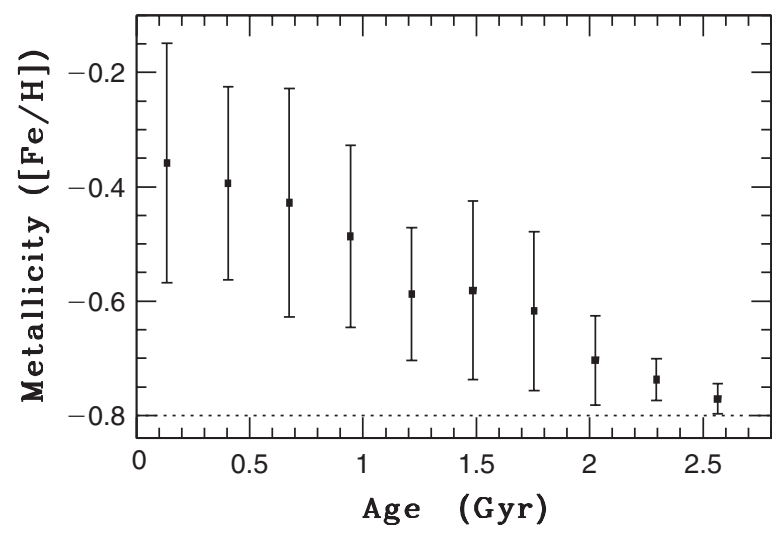

Figure 11 The AMR of new stars formed for the last $\sim 2.7 \mathrm{Gyr}$ evolution of the SMC in the fiducial model. The $1 \sigma$ dispersions in metallicities are shown by error bars for each of the age bins.

stars formed during the tidal interaction with the LMC and the Galaxy. However, the simulated $B$-band morphology is not so similar to the observed optical morphology, which has a 'wing' and a non-elliptical appearance in its central region. These apparent differences between the simulated and observed morphologies imply that the present models are not so successful in reproducing the $2 \mathrm{D}$ distributions of star-forming regions in the SMC.

Figure 10 shows that the line-of-sight velocity dispersion $(\sigma)$ profile for old stars has a strong radial gradient with the maximum $\sigma$ of $37 \mathrm{~km} \mathrm{~s}^{-1}$ and the mean $\sigma$ of $31 \mathrm{~km} \mathrm{~s}^{-1}$. The line-of-sight velocity profile $\left(V_{\mathrm{r}}-V_{\mathrm{r}, \mathrm{SMC}}\right)$ for old stars appears to have a small amount of rotation $\left(V<20 \mathrm{~km} \mathrm{~s}^{-1}\right)$. These simulated kinematic properties of the old stars are broadly consistent with the observed ones. $\sigma$ is systematically lower in new stars than in old ones, which reflects that the new stars originate from gas that can dissipate away the random kinematic energy produced during the SMC-LMC-Galaxy tidal interaction. New stars have a significant amount of rotation $\left(V \sim 40 \mathrm{~km} \mathrm{~s}^{-1}\right)$ and thus a high $V / \sigma(>1)$. These results suggest that young stellar populations with ages less than $\sim 3$ Gyr in the SMC can have kinematic properties significantly different from older ones such as PNe, carbon stars and red giants.

As shown in Figure 10, the line-of-sight velocity and velocity dispersion profiles of the gas are clearly different from those of the old stars in the sense that the gas show lower velocity dispersions $\left(7-42 \mathrm{~km} \mathrm{~s}^{-1}\right)$, a higher maximum rotational velocity $\left(\sim 49 \mathrm{~km} \mathrm{~s}^{-1}\right)$ and thus a higher $V / \sigma$ of $\sim 1.2$. The derived kinematic difference between old stars and gas and the high maximum rotational velocity are broadly consistent with the latest kinematic observations by $\mathrm{S} 04$. Figure 10 also shows that the velocity profiles for stellar and gaseous components are significantly asymmetric in the sense that the absolute magnitudes of the velocities appear to be systematically larger for $\alpha>1 \mathrm{~h}$. This asymmetry might well be due partly to the last LMC-SMC interaction about 0.2 Gyr ago. 


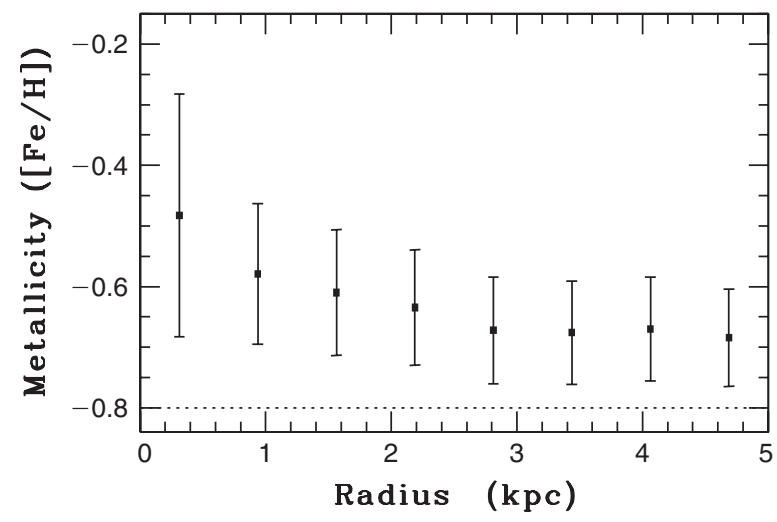

Figure 12 The radial dependence of the mean metallicities of new stars in the SMC for the fiducial model. The $1 \sigma$ dispersions in metallicities are shown by error bars for each of the radial bins.

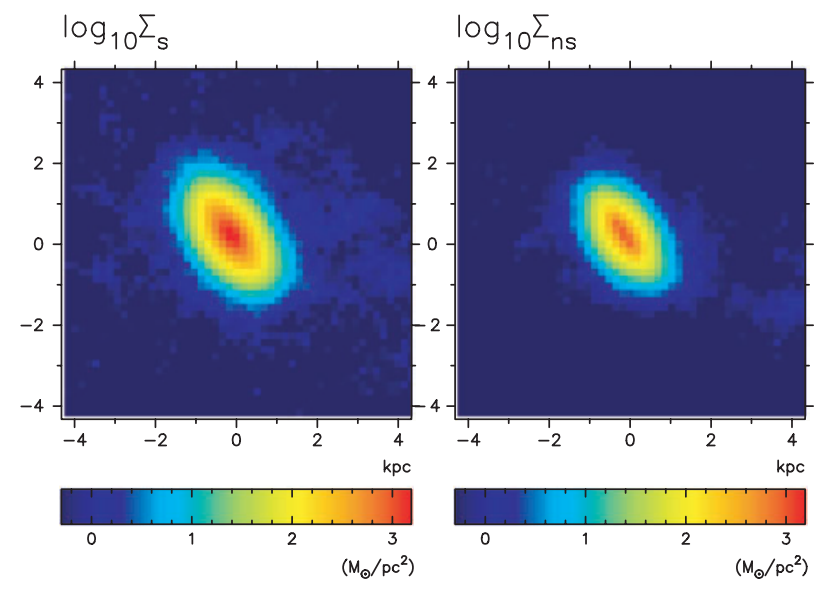

Figure 13 The same as Figure 6 but for the disk model G3 in which old stars are modeled as a rotating disk.

\subsubsection{Stellar Populations}

In this model with a relatively high $y_{\text {met }}=0.004$, the mean metallicity of the new stars evolves from $[\mathrm{Fe} / \mathrm{H}]=-0.75$ at $T=-2.2 \mathrm{Gyr}$ to $[\mathrm{Fe} / \mathrm{H}]=-0.56$ at $T=0 \mathrm{Gyr}$ (note that the initial gaseous metallicity is $[\mathrm{Fe} / \mathrm{H}]=-0.8)$. It should be stressed here that the final metallicity of new stars can be significantly smaller than the above value for smaller $y_{\text {met }}$ : the observed mean metallicity of young stars with ages less than $\sim 3$ Gyr can give constraints on the chemodynamical models of the SMC. As shown in Figure 11, there is an age-metallicityrelation (AMR) of new stars (i.e. more metal-rich for younger stars), though the dispersions in stellar metallicities appears to be quite large, especially for younger stars. The shape of the AMR and the metallicity dispersions also strongly depend on $y_{\text {met }}$, as discussed later in this paper.

Figure 12 shows that the mean metallicities of new stars are higher in the inner regions than in the outer ones for the central $3 \mathrm{kpc}$ of the simulated SMC. This negative metallicity gradient of new stars reflects the fact that chemically enriched gas due to star formation is transferred to the central region and converted into new stars there during the SMC-LMC-Galaxy interaction. The

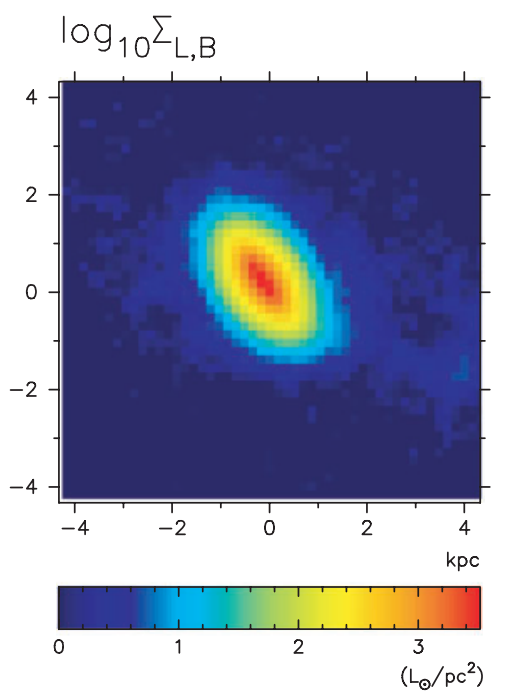

Figure 14 The same as Figure 9 but for the model G3.

metallicity dispersion is largest in the innermost radial bin, which implies that new stars currently in the central region originate from gas that are initially located in different regions with different metallicities. Although the negative metallicity gradient of new stars is a robust prediction of the present models, the slope of the gradient and the metallicity dispersions at radial bins depend strongly on $y_{\text {met }}$.

\subsection{Parameter Dependences}

Parameter dependencies of stellar and gaseous properties are summarized as follows.

(1) The final spatial distributions of stars do not depend strongly on model parameters in the dwarf spheroidal models: old and new stars have spherical and flattened distributions on the sky, respectively. The derived structural differences between old and new stars are broadly consistent with observations, which implies that the SMC was born as a dwarf spheroidal.

(2) The final structural and kinematic properties are quite different between the dwarf spheroidal and disk models. Figure 13 shows the final spatial distributions of old and new stars projected onto the sky for the disk model G3. The spatial distribution of old stars do not appear to be so spherical, which suggests that dynamical heating by the tidal fields of the LMC and the Galaxy is not strong enough to morphologically transform the initially disk-like shape into the more spherical one. New stars in the disk model show a bar in the projected distribution on the sky, which is consistent with the observed optical morphology of the SMC. Although only old stars appear to form the outer extended stellar halo, the spatial distributions between old and new stars are quite similar with each other. Figure 14 shows that the final $B$-band morphology in the disk model can be identified simply as an inclined disk galaxy. 


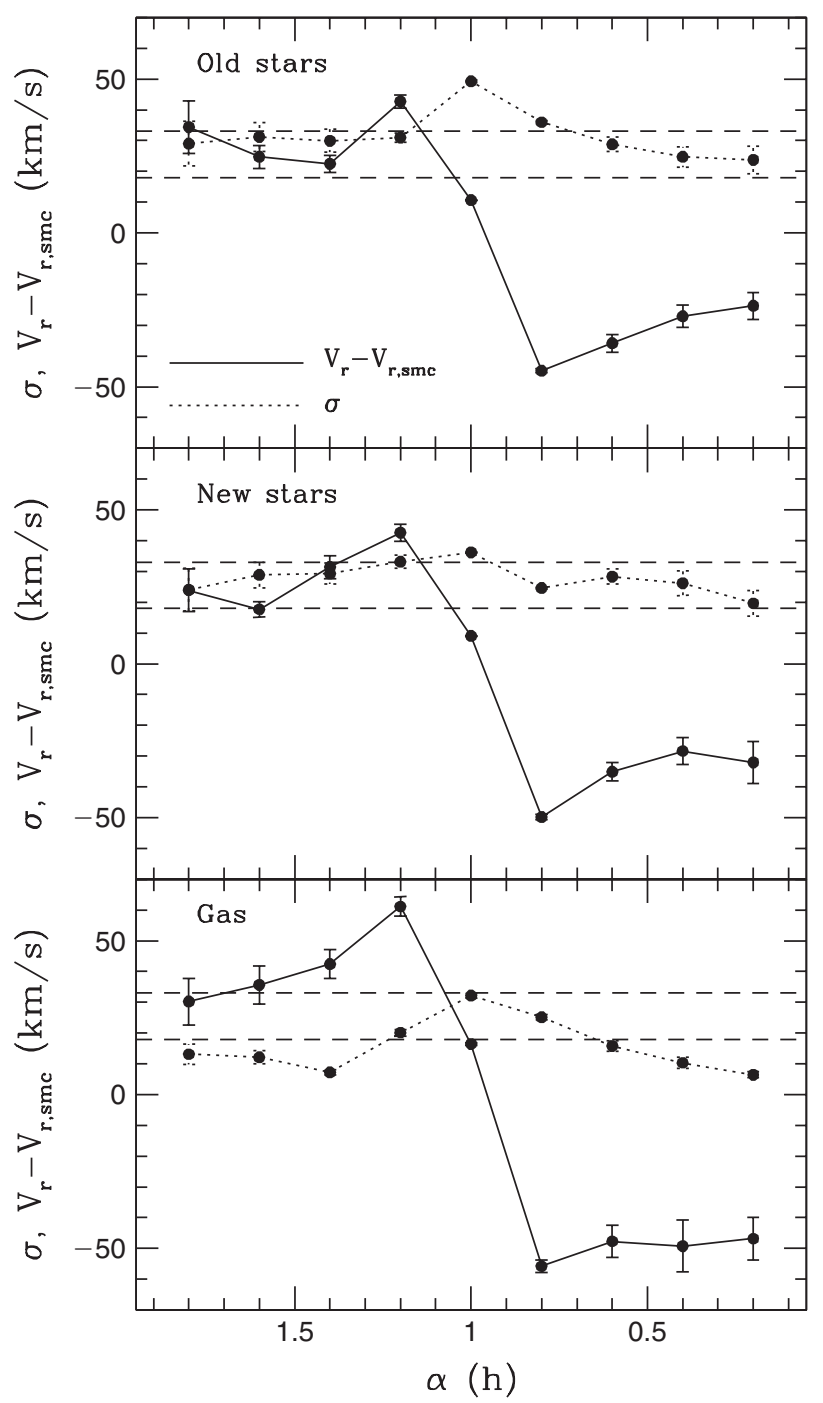

Figure 15 The same as Figure 10 but for the model G3.

(3) The final velocity dispersions $(\sigma)$ of old stars are roughly similar $\left(\sigma \sim 30 \mathrm{~km} \mathrm{~s}^{-1}\right)$ in different dwarf spheroidal models. The maximum line-of-sightvelocities $(V)$ of old stars are less than $20 \mathrm{~km} \mathrm{~s}^{-1}$ for most of the dwarf spheroidal models. It should be here stressed that the apparently non-negligible amount of rotation $\left(V<20 \mathrm{~km} \mathrm{~s}^{-1}\right)$ in old stars is due to the streaming motion of stars in the outer region of the SMC. The final velocity dispersions and maximum rotational velocities of new stars are systematically lower and higher, respectively, than those of the old ones, irrespective of model parameters.

(4) There are no significant kinematic differences between old stars, new ones and gas in the disk models, which is inconsistent with observations. Figure 15 shows that these three components show higher $V$ $\left(\sim 40 \mathrm{~km} \mathrm{~s}^{-1}\right)$ in the disk model G3 even after the strong tidal heating of the SMC by the LMC and the Galaxy. The velocity dispersions of new stars are only slightly smaller than those of old stars in these disk models. These results suggest that the disk models

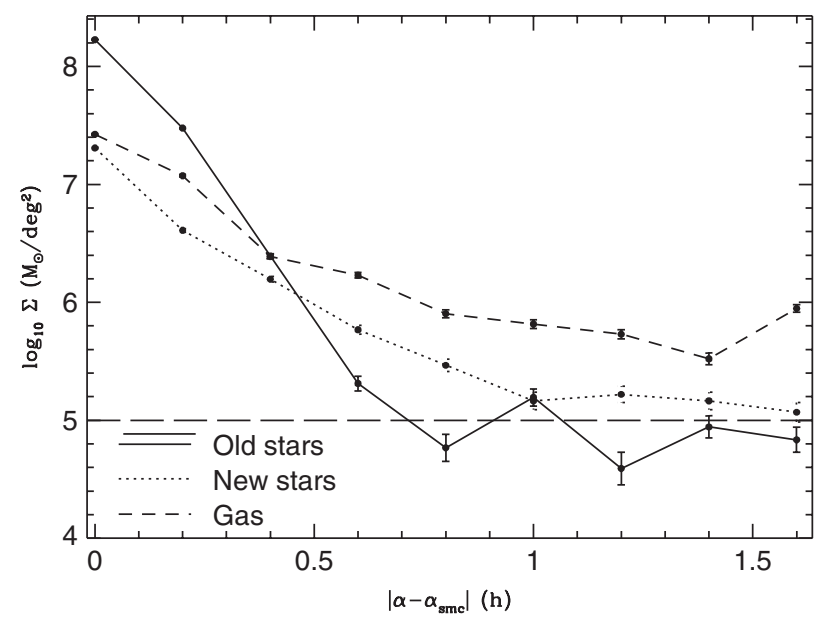

Figure 16 The same as Figure 7 but for the model G2 in which the initial spheroidal component is more compact than that in G1.

are less consistent with observations than the dwarf spheroidal ones in terms of kinematic properties of the SMC.

(5) The surface mass densities $(\Sigma)$ in the MB region depend strongly on initial distributions of stars of the SMC in the present models. Figure 16 shows an example of models in which $\Sigma$ of old stars can be significantly lower than those of new ones and gas. The model G2 shows that $\Sigma$ of old stars is lower than $10^{5} \mathrm{M}_{\odot} \mathrm{deg}^{-2}$ in some regions of the MB. This is mainly because a smaller number of old stars are tidally stripped in this model owing to the more compact initial distribution of the stars. These results imply that the observed $\Sigma$ of stars in the MB region can give strong constraints to the models for the initial distribution of old stars in the SMC.

(6) The SMC can lose a significant fraction (up to $\sim 40 \%$ depending on model parameters) of its initial mass in the present models. As a result of this, the final mass of the SMC can be significantly smaller than that suggested by observation $\left(2.4 \times 10^{9} \mathrm{M}_{\odot}\right.$ within the central $3 \mathrm{kpc}$ of the SMC, S04) for some models (e.g. G6, $\mathrm{C} 1$ and C3) with $M_{\mathrm{SMC}}=3.0 \times 10^{9} \mathrm{M}_{\odot}$. The initial sizes of the SMC $\left(R_{\mathrm{SMC}}\right)$ need to be quite small so that too efficient tidal stripping can be prevented in these low-mass models. Figure 17 shows that in the compact, low-mass model, the SMC loses only $26 \%$ of its initial mass so that the final mass within the central $5 \mathrm{kpc}\left(=2.1 \times 10^{9} \mathrm{M}_{\odot}\right)$ can be similar to the observed mass of the SMC (S04). The present models with $M_{\mathrm{SMC}}=3.0 \times 10^{9} \mathrm{M}_{\odot}$ and $R_{\mathrm{SMC}}=7.5 \mathrm{kpc}$ shows significantly lower final masses $\left(<2.0 \times 10^{9} \mathrm{M}_{\odot}\right)$, which suggests that the previous MS models in C06 may have serious problems in reproducing the present SMC mass. Figure 17 confirms that the Magellanic squall (i.e. gas transfer to the LMC from the SMC during the LMC-SMC interaction) can be seen in this low-mass SMC model. 


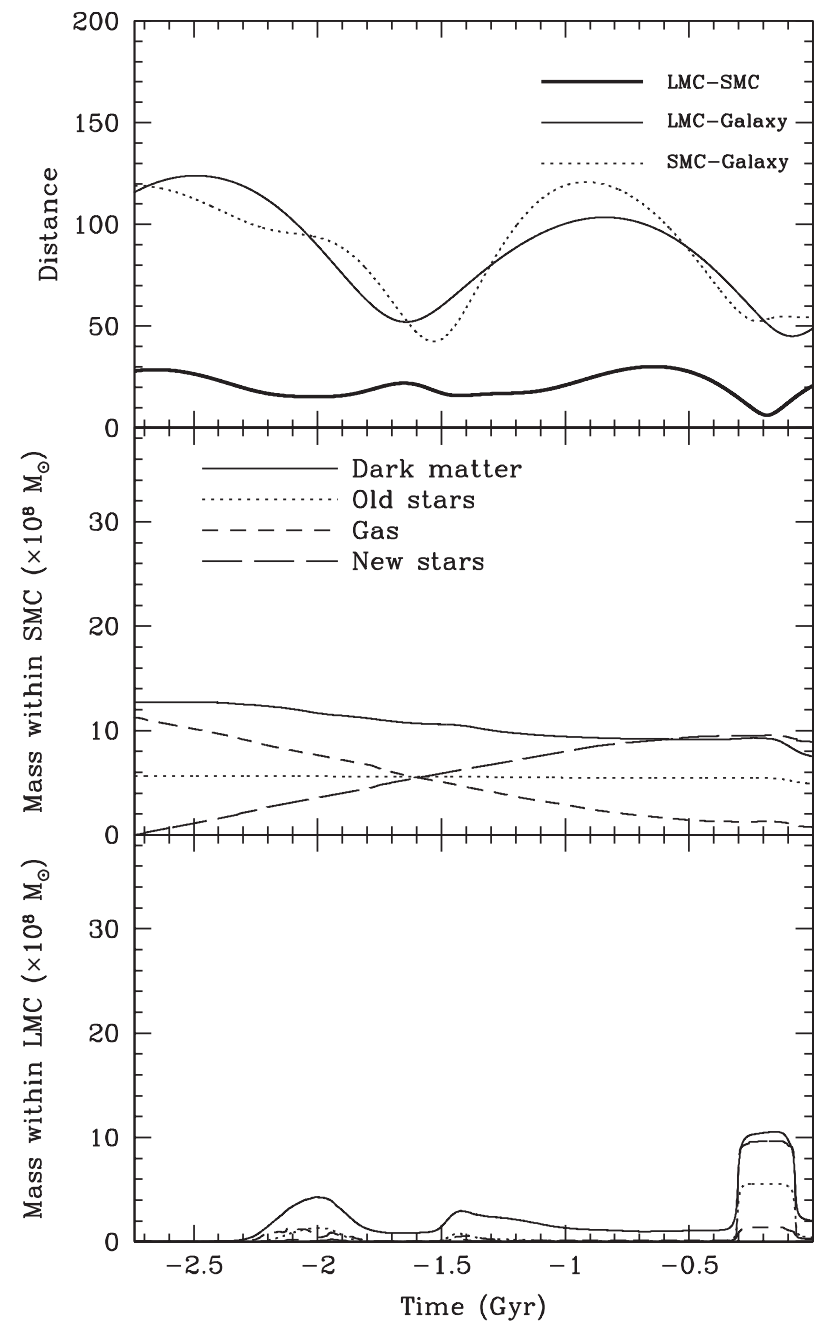

Figure 17 The same as Figure 4 but for the model G5 in which both the initial total mass and the size of the SMC are significantly smaller than that in G1.

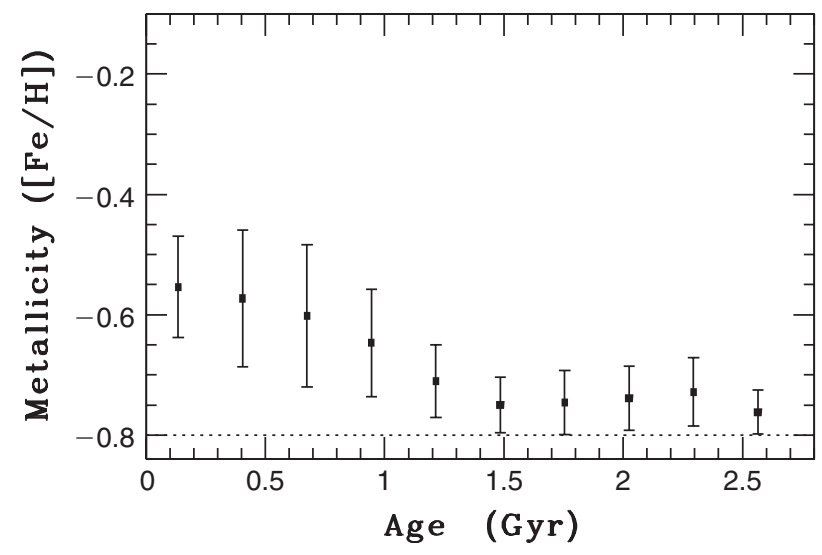

Figure 18 The same as Figure 11 but for the model G4 with $y_{\text {met }}=0.002$.

(7) The final mean stellar metallicity, the AMR of new stars and the radial stellar metallicity gradient in the SMC depend on $y_{\text {met }}$. For example, as shown in Figure 18 , the AMR in the model G4 with $y_{\text {met }}=0.002$ is significantly flatter than that in G1. Both the stellar metallicity for young stars with ages less than $0.2 \mathrm{Gyr}$ and the dispersions in stellar metallicities in this model are significantly smaller than those in G1. It is, however, currently unclear which of the two models, G1 and G4, is more consistent with observations on abundances of young stars with ages less than 3 Gyr.

\section{Discussion}

\subsection{Origin of Stellar and Gaseous Kinematics in the $S M C$}

Previous kinematic studies based on radial velocities of older stellar populations such as $\mathrm{PNe}$ and red giants suggested that the stellar component of the SMC has the velocity dispersion of $20-30 \mathrm{~km} \mathrm{~s}^{-1}$ and appears to have no global rotation (e.g. Dopita et al. 1995; Suntzeff et al. 1986; Hatzidimitriou et al. 1993). The latest survey of 2046 red giant stars have suggested that the stellar component of the SMC is primarily supported by its velocity dispersion (Harris \& Zaristky 2006). Observational studies of Hi gas, on the other hand, showed that the gaseous component has global rotation with the peak rotational velocity of about $60 \mathrm{~km} \mathrm{~s}^{-1}$ (S04). No theoretical studies, however, have yet provided a physical explanation for this intriguing kinematic difference between stellar and gaseous components in the SMC.

The present study has first shown that kinematic differences between old stars and gas can be clearly seen only in the dwarf spheroidal models: both stellar and gaseous components of the disk models show significant rotation even after strong tidal heating by the LMC and the Galaxy and have no kinematic differences between them. These results imply that the SMC previously did not have a rotating disk composed both of older stars and gas but had an extended gas disk kinematically decoupled from its stellar component. The rotating gaseous disk is one of essential ingredients in the previous successful models for the MS (GN96, YN and C06). Therefore the formation processes of the MS described in these studies would not be changed significantly if stellar spheroids rather than stellar disks were adopted in these studies. The previous collisionless models (BC07b) and the present ones in which the SMC is assumed to be a dwarf spheroidal can reproduce physical properties of the MS reasonably well (see Appendix A for more discussions on the formation of the MS).

The present study suggests that younger stars formed from the rotating gas disk of the SMC for the last $\sim 2.7 \mathrm{Gyr}$ can show a smaller (projected) central velocity dispersion $\left(\sigma \sim 20 \mathrm{~km} \mathrm{~s}^{-1}\right)$ and a larger (projected) rotational velocity $\left(V \sim 40 \mathrm{~km} \mathrm{~s}^{-1}\right)$. Although 3D structures of Cepheid variables (possible candidates of younger stars) along the line-of-sight were previously investigated for the SMC (e.g. Caldwell \& Coulson 1986), kinematic properties of young stellar populations are largely unknown. It is thus doubtless worthwhile for future observations to confirm whether or not younger stellar populations with ages less 
than 3 Gyr in the SMC show rotational kinematics, unlike its older stellar populations.

\subsection{Formation of a Dwarf Spheroidal with an Extended HI Disk}

The present study has first shown that the observed dynamical properties of the SMC can be successfully reproduced by the new dwarf spheroidal models. The previous disk models which have been often adopted in explaining the MS (e.g. YN03) would not be reasonable. We therefore suggest that the SMC before interacting strongly with the LMC and the Galaxy (i.e. more than $3 \mathrm{Gyr}$ ago) was a gas-rich dwarf like the NGC 6822 with both a spheroidal component composed of old and intermediate-age stars and an extended Hi disk (e.g. de Blok \& Walter 2003). If the SMC was really a gas-rich dwarf spheroidal, then the following question arises: how were the main body of the central spheroid and the outer Hi gas disk formed?

Mayer et al. (2001) showed that low-luminosity disk galaxies in the Local Group can be transformed into dwarf spheroidal and elliptical galaxies, if they are strongly influenced by tidal fields of the giant galaxies like the Galaxy. Since this transformation scenario predicts quite effective tidal stripping of gas and stars in the outer parts of disks, it would not be possible that the transformed dwarf spheroids can still contain the outer HI disk. Therefore the formation of the SMC with an extended Hi disk would not result from past tidal interaction of the SMC with the Galaxy.

Recent HI observations of the SMC have suggested that the total mass of the SMC derived from the HI rotation curve is about $2.4 \times 10^{9} \mathrm{M}_{\odot}$ and thus consistent with a dynamical model without a dark-matter halo (e.g. S04). These observations may well imply that the SMC is a 'tidal dwarf' which can be formed from tidal tails during strong tidal interaction between two galaxies (e.g. Duc et al. 2000), because tidal dwarfs can not contain dark matter components that are initially in halos of their host galaxies. Although there are two luminous galaxies (i.e. M31 and the Galaxy) in the Local Group which can be host galaxies for tidal dwarfs, there appears to be no observational evidence for their past strong interaction. Thus the tidal dwarf scenario currently appears to be less promising as a SMC-formation model.

Thus we suggest an 'accretion scenario' in which the SMC is likely to have developed its spheroidal component at the epoch of galaxy formation and then gradually formed its extended Hi disk via gaseous accretion from its outer halo. The potential problem of this accretion scenario is that the strong tidal field of the LMC and the Galaxy could not have allowed the halo gas of the SMC to be accreted onto the disk of the SMC, if the SMC has been bound closely to the LMC and the Galaxy since its formation. The present tidal radius of the present SMC is only $\sim 5.0-7.5 \mathrm{kpc}$ (GN96; YN; C06), which implies that only a small amount of gas could have been accreted onto the SMC without being stripped by the LMC and the Galaxy. The present distances of the SMC with respect to the LMC and the Galaxy may have been significantly larger in the past than in the present one. If this is the case, the SMC may have developed its massive Hi disk through gradual accretion from the halo to finally become a dwarf spheroidal with an extended Hi disk. Other scenarios (e.g. major merging) for the formation of the SMC are discussed in detail by Bekki \& Chiba (2008).

In the above discussions, we assume that the SMC has a dynamically hot spheroid with no/little rotation. It is, however, possible that the SMC is actually a rotationally flattened spheroid viewed almost face-on (i.e. along the rotational axis): we can not detect rotation of the spheroid in the line-of-sight-velocity field, even if the old stars of the SMC have rotational kinematics. If this is the case, the SMC is almost like a polar-ring galaxy in which the rotational axes of stars and gas are perpendicular with each other. Although this possibility can not be ruled out by current observations, the probability of such a configuration of the SMC with respect to the Galaxy would be very low. Thus we suggest that the observed apparent differences in kinematics between old stars and $\mathrm{HI}$ gas in the SMC reflect real differences in internal kinematics between them. Such differences may well suggest that the SMC would have experienced accretion/merging event of very gas-rich objects (e.g. gas-rich dwarfs) in its dynamical history possibly in a small group where galaxy merging is more likely.

\subsection{On the Possible Absence of Older Stars in the MB}

Observational studies revealed a number of intriguing physical properties of stars and gas in the inter-Cloud regions and the MB (e.g. Irwin, Demers \& Kunkel 1990; Muller et al. 2004; Mizuno et al. 2006; Harris 2007; Kato et al. 2007; Nishiyama et al. 2007). Muller et al. (2004) reported that the MB is composed of two kinematically and morphologically distinct structures and shows a remarkable velocity offset around $\delta=-73.5^{\circ}$ in the $\delta-V_{\text {rad }}$ plane. The discovery of molecular clouds (Mizuno et al. 2006) and the possible detection of pre-mainsequence stars (Nishiyama et al. 2007) in the MB strongly suggest that star formation in the MB is currently ongoing and possibly triggered by the last LMC-SMC interaction. Harris (2007) did not detect an older stellar population in the MB and thus suggested that the MB originates purely from gaseous components of the MCs. Since the observed structural and kinematic properties of the MB and the possible physical mechanisms for star formation in the MB have been already discussed by Muller \& Bekki (2007) and Bekki \& Chiba (2007), respectively, we here focus on the origin of the possible absence of older stellar populations in the MB.

The present model with the GN96 velocity type and $M_{\mathrm{smc}, \mathrm{s}} \sim 10^{9} \mathrm{M}_{\odot}$ predicts that the projected surface mass density in the $\mathrm{MB}$ is roughly $6 \times 10^{4}$ to $3.0 \times 10^{6} \mathrm{M}_{\odot} \mathrm{deg}^{-2}$. The presence of old stars in the MB is due largely to the tidal stripping of stars during the last LMC-SMC close encounter with its pericenter distance of $\sim 8 \mathrm{kpc}$ about $0.2 \mathrm{Gyr}$ ago. The presence of old stars in the 
MB thus appears to be inconsistent with observations by Harris (2007), though the observational upper limit of the mass density of an older stellar population is not clearly shown in Harris (2007). The following two are the possible interpretations for this apparent inconsistency between the simulations and the observations. The first is that the simulated last LMC-SMC interaction needs to be weaker, so that fewer old stars end up in the MB. The second is that the initial size of the stellar spheroid (or disk) in the simulation needs to be smaller to prevent its efficient tidal stripping by the LMC.

Noël \& Gallart (2007) have recently found the presence of intermediate-age and old stars in the southern regions at $6.5 \mathrm{kpc}$ from the SMC center. This result, which is in a striking contrast to the results by Harris (2007), suggests that the SMC has an outer stellar halo component and is possibly more massive than other observations have ever suggested. Furthermore, this result possibly implies that there could be older stars in the MB, if the stellar halo of the SMC has a spherical distribution. The upper limit for the projected mass density of older stars in the $\mathrm{MB}$ is crucial to determine whether the last LMC-SMC interaction is the main mechanism of the MB formation. We thus suggest that more extensive observational studies need to be done to provide strong constraints for the formation models of the MB.

If the projected surface density of older stars in the MB is truly low, as the result by Harris (2007) suggests, the result by Noël \& Gallart (2007) possibly implies that the stellar halo of the SMC is quite asymmetric and elongated to the north-south direction. The strong tidal fields of the LMC and the Galaxy can transform the outer shape of the SMC's stellar halo so that the recent interaction history, which is determined by the orbits of the MCs, can be imprinted on the shape of the stellar halo. We thus suggest that the direction of the major axis of the possibly elongated stellar halo might well provide some constraints on the orbit of the SMC around the LMC: the direction would be perpendicular to the orbit of the SMC and point to the center of the LMC (thus can constrain the orbital plane of the LMC-SMC system).

\subsection{Dark-Matter Properties of the SMC}

We have first pointed out that (1) the dark-matter halo of the SMC is highly likely to have a very large core $(>5 \mathrm{kpc})$ and (2) the observed rotation curve of $\mathrm{HI}$ and the total mass of the SMC within the central few kpc can be explained better by the SB profile of the dark matter distribution. The SB profile with a large core might well explain why the SMC appears to have no dark matter in the central few kpc (S04), though a fully self-consistent dynamical model including the dark-matter halo has not been construed yet for the SMC (Bekki \& Chiba, in preparation). If the SMC has a such large, low-density core of its dark-matter halo, it is highly likely that the halo was initially quite extended and therefore lost a substantial amount of its original mass owing to the past tidal interaction with the LMC and the Galaxy since its formation.
As shown in the present simulations, significant fractions of original masses in the SMC (up to $\sim 40 \%$ ) can be lost during the last 2.7 Gyr of LMC-SMC-Galaxy interaction. We have therefore suggested that in order to explain the observed total mass of the SMC within the central $3 \mathrm{kpc}$, the original mass of the SMC should be significantly more massive than $M_{\mathrm{SMC}}=3.0 \times 10^{9} \mathrm{M}_{\odot}$, which is adopted in previous MS models (GN96 and C06). We have demonstrated that the models with $M_{\mathrm{SMC}}=4.5 \times 10^{9} \mathrm{M}_{\odot}$ can better explain the present SMC mass even after the significant mass loss of the SMC due to the interaction. It should be noted here that such models with larger $M_{\text {SMC }}$ and thus larger Hi gas masses can naturally solve the problems of the previous MS models in explaining the observed total mass of the MS and the LAs (e.g. YN03). We thus suggest that the SMC initially has a larger total mass (possibly as much as $4.5 \times 10^{9} \mathrm{M}_{\odot}$ ) and a large core in the dark matter distribution.

\section{Conclusions}

We have investigated structural, kinematic and chemical properties of stars and gas in the SMC interacting with the LMC and the Galaxy based on chemodynamical simulations. We have adopted a new dwarf spheroidal model with an extended HI gas disk for the SMC and investigated the chemical and dynamical evolution and the star formation history of the SMC for the last $\sim 2.7 \mathrm{Gyr}$. The main results are summarized as follows.

(1) The final spatial distribution of the old stars projected onto the sky is more spherical even after the strong LMC-SMC-Galaxy interaction whereas that of the new stars is significantly flattened and appears to be a bar. This difference in spatial distributions between old and new stars is due to the fact that new stars are formed in the thin Hi disk of the SMC during the tidal interaction. These result suggest that the observed 'bar' is mostly composed of new stars recently formed in the SMC. Some fraction of the old stars initially in the SMC is stripped to form the outer stellar halo of the SMC. The distribution of the stellar halo projected onto the sky appears to be slightly asymmetric in the north-south direction, which possibly reflects the strong tidal interaction with the LMC about $0.2 \mathrm{Gyr}$ ago.

(2) Old stars have a line-of-sight velocity dispersions, $\sigma \sim 30 \mathrm{~km} \mathrm{~s}^{-1}$, weak rotation, with the maximum rotational velocity of $V<20 \mathrm{~km} \mathrm{~s}^{-1}$ and show asymmetry in their profiles. New stars have a smaller $\sigma$ than old ones and a significant amount of rotation $(V / \sigma>1)$. These kinematic differences between old and new stars do not depend on model parameters. It should be stressed that the derived $V$ of old stars is highly likely to be overestimated by the stream motions of the stars along the tidal tails of the SMC.

(3) The HI gas shows velocity dispersions of $\sigma \sim 10$ $40 \mathrm{~km} \mathrm{~s}^{-1}$ depending on the distances from the 
SMC's center, a high maximum rotational velocity $\left(V \sim 50 \mathrm{~km} \mathrm{~s}^{-1}\right)$ and a spatial distribution similar to that of new stars. The derived higher velocity dispersion of gas is due to the tidal disturbance of gas by the LMC and the Galaxy.

(4) The mean metallicity of stars can increase from $[\mathrm{Fe} / \mathrm{H}] \sim-0.80$ to $[\mathrm{Fe} / \mathrm{H}] \sim-0.56$ for $y_{\text {met }}=0.004$. The stellar populations show a weak AMR in the sense that younger stars are more likely to be more metal-rich. The final stellar $[\mathrm{Fe} / \mathrm{H}]$ and the AMR depend strongly on $y_{\text {met }}$ (e.g. the final metallicity is $[\mathrm{Fe} / \mathrm{H}] \sim-0.66$ for $\left.y_{\text {met }}=0.002\right)$. More metal-rich stars are more likely to be located in the inner regions of the SMC, irrespective of model parameters.

(5) Although the MB can be formed in most models, the simulated MB inevitably contains old stars. This is mainly because some fraction of old stars initially in the spheroidal component of the SMC is tidally stripped to be distributed in the MB region. The mean surface density of stars in the MB is different in different models and ranges from $\sim 6 \times 10^{4} \mathrm{M}_{\odot} \mathrm{deg}^{-2}$ to $\sim 3 \times 10^{6} \mathrm{M}_{\odot} \mathrm{deg}^{-2}$. If these numbers are inconsistent with observations, these results mean that the orbital evolution of the MCs for the last $0.2 \mathrm{Gyr}$ in our models needs to be significantly modified so as to reduce significantly the stellar mass in the MB.

(6) The disk model shows flattened spatial distributions and higher maximum rotational velocities $\left(V>40 \mathrm{~km} \mathrm{~s}^{-1}\right)$ in old stars, which appear to be inconsistent with observations. Thus the dwarf spheroidal model can better explain observations than the disk one for a given orbit of the SMC in terms of structures and kinematics of old stars in the SMC. Chemical enrichment processes are not so different between the dwarf and the disk models.

(7) The simulated MS, which is composed mostly of HI gas, shows bifurcation in its structure and kinematics. The models based on the GN96 velocity type can much better explain distributions and kinematics of $\mathrm{HI}$ gas in the MB than those based on the K06 one that are derived from the latest HST proper motion measurements. Since we did not investigate the MS formation for all of the possible unbound orbits of the MCs, it is unclear whether all of the unbound orbits for the MCs consistent with K06 are highly unlikely to explain the MS formation. These results imply that the physical interpretations of the HST proper motion measurements need to be reconsidered in the context of the formation of the MS. We have suggested that future abundance studies of the MS from QSO absorption lines can provide valuable information on formation processes and early chemical evolution of the outer $\mathrm{HI}$ disk of the SMC.

(8) The SMC is suggested to initially have a large core $(>5 \mathrm{kpc})$ in its dark matter distribution and a larger total mass $\left(M_{\mathrm{SMC}}>3.0 \times 10^{9} \mathrm{M}_{\odot}\right)$ so that the observed kinematic properties of $\mathrm{HI}$ in the present SMC can be better explained. The more massive SMC with a low-density dark-matter halo is suggested to explain better observations of the inner profile of the $\mathrm{HI}$ rotation curve and of the total gas mass in the MS and the LAs.

The present study adopted the 'classical' orbits of the MCs (GN96) rather than the new one (K06) in order to compare results of the present new SMC model with those of the previous ones (e.g. Yoshizawa \& Noguchi 2003; C06) that also adopted the classical orbits. We thus have not solved the problems related to the inconsistency between the predicted velocities of the MCs and the observed ones (K06). In our forthcoming papers, we discuss how to solve the problems based on more sophisticated numerical simulations.

\section{Acknowledgments}

We are grateful to the anonymous referee for valuable comments, which contribute to improve the present paper. The numerical simulations reported here were carried out on GRAPE systems kindly made available by the Astronomical Data Analysis Center (ADAC) at National Astronomical Observatory of Japan (NAOJ). K.B. acknowledges financial support from the Australian Research Council (ARC) and ADAC throughout the course of this work.

\section{References}

Andredakis, Y. C. \& Sanders, R. H., 1994, MNRAS, 267, 283

Bekki, K., 1998, ApJ, 502, L133

Bekki, K., 2007, MNRAS, 380, 1669

Bekki, K., Couch, W. J., Beasley, M. A., Forbes, D. A., Chiba, M. \& Da Costa, G., 2004, ApJ, 610, L93

Bekki, K. \& Chiba, M., 2005, MNRAS, 356, 680

Bekki, K. \& Chiba, M., 2007, MNRAS, 381, L16

Bekki, K. \& Chiba, M., 2006, ApJ, 637, L97

Bekki, K. \& Chiba, M., 2008, ApJ, 679, L89

Bekki, K., Chiba, M. \& McClure-Griffiths, N. M., 2008, ApJ, 672, L17

Bekki, K. \& Stanimirović, S., 2009, MNRAS, submitted (arXiv/0807.2102v1)

Besla, G., Kallivayalil, N., Hernquist, L., Robertson, B., Cox, T. J., van der Marel, R. P. \& Alcock, C., 2007, ApJ, 668, 949

Binney, J. \& Tremaine, S., 1987, Galactic Dynamics (Princeton: Princeton Univ. Press)

Broeils, A. H. \& van Woerden, H., 1994, A\&AS, 107, 129

Brüns, C. et al., 2005, A\&A, 432, 45

Burkert, A., 1995, ApJ, 447, L25

Caldwell, J. A. R. \& Coulson, I. M., 1986, MNRAS, 218, 223

Cioni, M.-R. L., Girardi, L., Marigo, P. \& Habing, H. J., 2006, A\&A, 448,77

Connors, T. W., Kawata, D. \& Gibson, B. K., 2006, MNRAS, 371, 108

de Blok, W. J. G. \& Walter, F., 2003, MNRAS, 341, L39

Dopita, M. A., Lawrence, C. J., Ford, H. C. \& Webster, B. L., 1985, ApJ, 296, 390

Duc, P.-A., Brinks, E., Springel, V., Pichardo, B., Weilbacher, P. \& Mirabel, I. F., 2000, AJ, 120, 1238

Gardiner, L. T. \& Noguchi, M., 1996, MNRAS, 278, 191

Hardy, E., Suntzeff, N. B. \& Azzopardi, M., 1989, ApJ, 344, 210

Harris, J. \& Zaritsky, D., 2004, AJ, 127, 1531

Harris, J. \& Zaritsky, D., 2006, AJ, 131, 2514

Harris, J., 2007, ApJ, 658, 345 
Hatzidimitriou, D., Croke, B. F., Morgan, D. H. \& Cannon, R. D., 1997, A\&AS, 122, 507

Hernquist, L. \& Katz, N., 1989, ApJS, 70, 419

Hunter, D. A., Elmegreen, B. G. \& Baker, A. L., 1998, ApJ, 493, 595 Ichikawa, S. I., Wakamatsu, K. I. \& Okamura, S., 1986, ApJS, 60, 475 Idiart, T. P., Maciel, W. J. \& Costa, R. D. D., 2007, A\&A, 472, 101 Irwin, M. J., Demers, S. \& Kunkel, W. E., 1990, AJ, 99, 191

Kallivayalil, N., van der Marel, R. P. \& Alcock, C., 2006, ApJ, 652, 1213

Kato, D. et al., 2007, PASJ, 59, 615

Kennicutt, R. C., Jr., 1998, ARA\&A, 36, 189

Mayer, L. et al., 2001, ApJ, 559, 754

Monaghan, J. J. \& Lattanzio, J. C., 1985, A\&A, 149, 135

Mastropietro, C., Moore, B., Mayer, L., Wadsley, J. \& Stadel, J., 2005, MNRAS, 363, 509

Mizuno, N. et al., 2006, ApJ, 643, L107

Muller, E., Stanimirović, S., Rosolowsky, E. \& Staveley-Smith, L., 2004, ApJ, 616, 845

Muller, E. \& Bekki, K., 2007, 381, L11

Muñoz, R. R. et al., 2006, ApJ, 649, 201

Murai, T. \& Fujimoto, M., 1980, PASJ, 32, 581

Navarro, J. F., Frenk, C. S. \& White, S. D. M., 1996, ApJ, 462, 563

Nidever, D. L., Majewski, S. R. \& Butler B. W., 2007, ApJ, 679, 432

Nishiyama, S. et al., 2007, ApJ, 658, 358

Noël, N. E. D. \& Gallart, C., 2007, ApJ, 665, 23

Noguchi, M., 1988, A\&A, 203, 259

Pagel, B. E. J. \& Tautvaisiene, G., 1998, MNRAS, 299, 535

Piatek, S., Pryor, C. \& Olszewski, E. W., 2008, AJ, 135, 1024
Piatti, A. E., Sarajedini, A., Geisler, D., Clark, D. \& Seguel, J., 2007, MNRAS, 377, 300

Putman, M. E. et al., 1998, Natur, 394, 752

Russell, S. C. \& Dopita, M. A., 1992, ApJ, 384, 508

Salucci, P. \& Burkert, A., 2000, ApJ, 537, L9

Shattow, G. \& Loeb, A., 2009, MNRAS, 392, 21

Stanimirović, S., Staveley-Smith, L. \& Jones, P. A., 2004, ApJ, 604,176

Schmidt, M., 1959, ApJ, 129, 243

Spite, M., Huille, S., Spite, F. \& Francois, P., 1988, A\&AS, 76, 405

Sugimoto, D., Chikada, Y., Makino, J., Ito, T., Ebisuzaki, T. \& Umemura, M., 1990, Natur, 345, 33

Suntzeff, N. B., Friel, E., Klemola, A., Kraft, R. P. \& Graham, J. A., 1986, AJ, 91, 275

Thornton, K., Gaudlitz, M., Janka, H.-Th. \& Steinmetz, M., 1998, ApJ, 500, 95

Torres, G. \& Carranza, G. J., 1987, MNRAS, 226, 513

Tsujimoto, T., Nomoto, K., Yoshii, Y., Hashimoto, M., Yanagida, S. \& Thielemann, F.-K., 1995, MNRAS, 277, 945

van den Bergh, S., 2000, The Galaxies of the Local Group (Cambridge: Cambridge Univ. Press)

Vazdekis, A., Casuso, E., Peletier, R. F. \& Beckman, J. E., 1996, ApJS, 106, 307

Westerlund, B. E., 1997, The Magellanic Clouds (Cambridge: Cambridge Univ. Press)

Widrow, L. M. \& Dubinski, J., 2005, ApJ, 631, 838

Yoshizawa, A. \& Noguchi, M., 2003, MNRAS, 339, 1135 


\section{Appendix A Formation of the MS}

Previous tidal models of the MS successfully reproduced a number of observed physical properties of the MS, such as the presence of the LA, the absence of stars in the MS and bifurcated structures and kinematics of the MS (e.g. Murai \& Fujimoto 1980; GN96; YN; C06). Although the ram pressure models, in which the MS originates from the gas disk of the LMC, provided some explanations for the origin of the observed kinematic properties of the MS, they failed to explain the presence of the LA and the bifurcated structure of the MS (e.g. Mastropietro et al. 2005). Given that the simple version of the tidal models has some difficulties in explaining in the location of the LA on the sky (Bekki, Chiba \& McClure-Griffiths 2008), it would be fair to claim that it remains unclear whether the tidal interaction between the MCs and the Galaxy is the dominant formation mechanism for the MS. Also it is unclear whether the present new dwarf spheroidal models can explain physical properties of the MS as well as the previous ones.

Recent observational studies based on proper motion measurements of the MCs by HST ACS have shed new light on the above unresolved problem of the MS formation (K06, Besla et al. 2007). Based on a systematical study of orbital evolution of the MCs, Besla et al. (2007) suggested that the MCs arrived at the Galaxy about $0.2 \mathrm{Gyr}$ ago for the first time in their histories, if the observed proper motions of the MCs are correct. These studies thus imply that the MS is unlikely to be formed as a result of the past tidal interaction of the SMC with the LMC and the Galaxy about $1.5 \mathrm{Gyr}$ ago. It is, however, unclear whether theoretical models with the orbits of the MCs being consistent with observations by K06 can reproduce the observed properties of the MS.

Thus, we need to investigate both whether the observed MS can be well reproduced by the present new SMC models with the GN96 velocity type and whether the present models with the K06 one can explain the observed properties of the MS as well. We show the results of the two collisionless models ( $\mathrm{C} 1$ and $\mathrm{C} 2$ ), because other models show similar properties to those of these two models. The detailed results of the MS formation in more selfconsistent $\mathrm{N}$-body models for the MCs and the Galaxy will be described in Bekki \& Chiba (in preparation).

Figure 19 shows that the locations of the MS and the LAs in the model $\mathrm{C} 1$ are broadly consistent with the observed ones (e.g. Putman et al. 1998), though the shapes of the LAs appears to be too broadened. The bifurcation of the MS can be clearly seen, which confirm the early results by C06. Figure 20 clearly shows the velocity gradient of the MS along the Magellanic longitudes, which is consistent with observations. Figure 20 also shows the bifurcation in kinematics (i.e. $V_{\mathrm{LSR}}$ ) for the Magellanic longitude larger than 50 degrees. These simulated bifurcation in the structural and kinematic properties of the MS will soon be compared with the corresponding observations (e.g. Brüns et al. 2005; Nidever, Majewski \& Butler 2007) so that the validity of the models will be addressed.

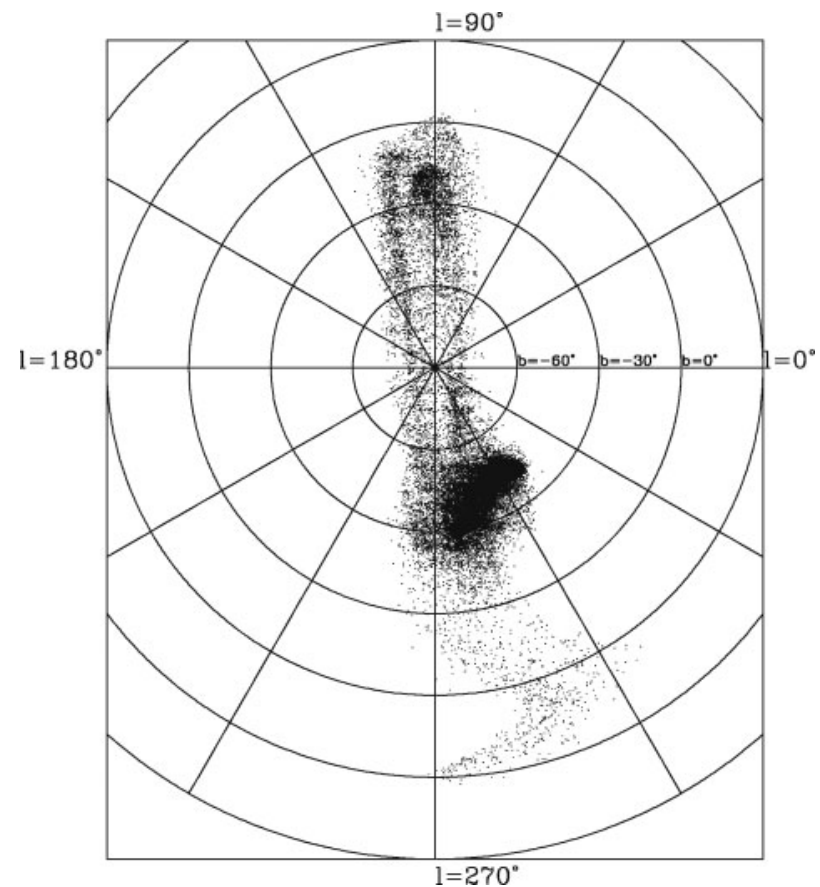

Figure 19 The final distributions of gas in the galactic coordinate system $(l . b)$ for the dwarf spheroidal model $\mathrm{C} 1$. This model is designed to compare with the early results obtained by the collisionless MS models of GN96 and C06 in which the SMC is modeled as a disk galaxy.

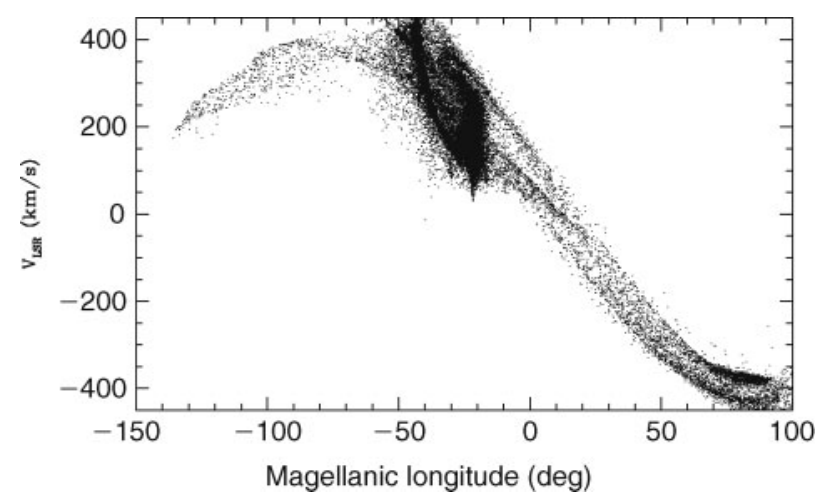

Figure $20 V_{\text {LSR }}$ (local-standard-of-rest velocities) of gaseous particles as a function of Magellanic longitude for the model $\mathrm{C} 1$.

Figure 21 shows that the simulated distributions of gas on the sky are inconsistent with observation in the model C2 with the K06 velocity type, though two gaseous streams can be formed from the extended gas disk of the SMC as a result of SMC-Galaxy interaction. It is also confirmed in the present study that the observed locations of the MS and the LA are not reproduced by the present models with the K06 velocity types irrespective of model parameters (e.g. $M_{\mathrm{SMC}}$ ). There can be mainly two interpretations of the above results. The first is that the although the 3D space motions of the MCs derived from the observed proper motions of the MCs are very close to the true ones, the adopted assumption that the MS originates from the SMC is wrong. Besla et al. (2007) proposed a scenario in 


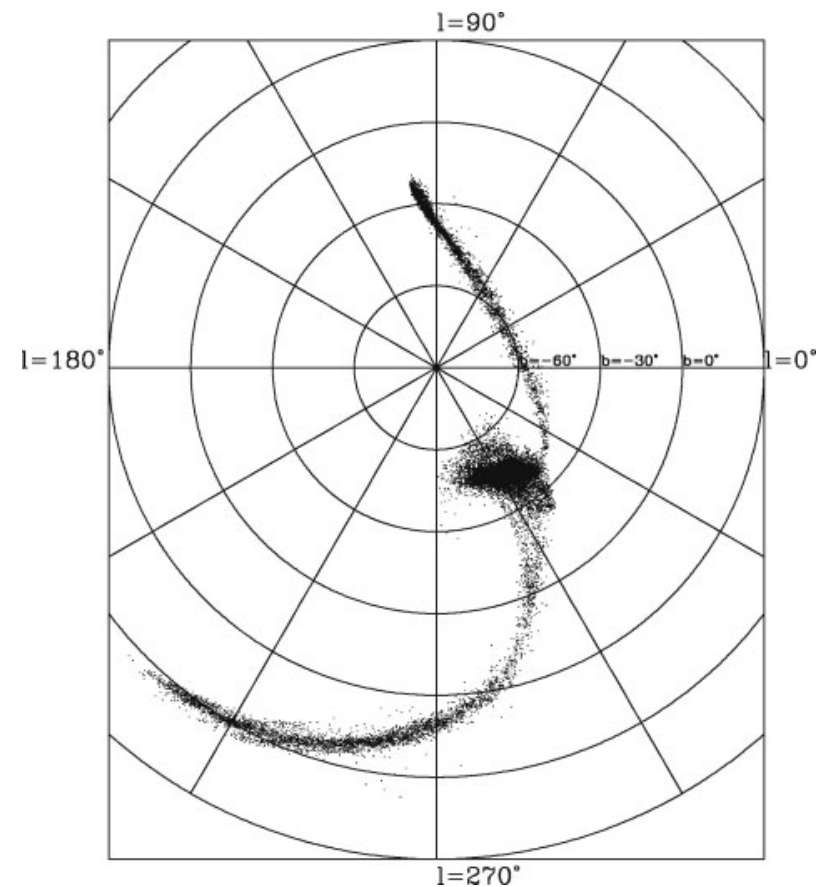

Figure 21 The same as Figure 19 but for the model C2.

which the MS originates from gaseous components initially within the LMC possibly through gaseous outflow triggered by stellar feedback effects. Owing to the lack of numerical studies based on the above scenario, it remains unclear whether the MS can originate in the LMC, if the 3D space motions of the MCs proposed by K06 are correct.

The second is that the $3 \mathrm{D}$ space motions of the MCs proposed by $\mathrm{K} 06$ are not close to the true ones, so that the inconsistency in the 3D space motions between the tidal models (e.g. GN96) and the observationally suggested ones (K06) cannot be a serious problem for the tidal models. Bekki (2008) first showed that the mean proper motion of the SMC derived from the three local fields can be significantly different from that of the center of the mass for the SMC, so that the 3D proper motion of the $\mathrm{SMC}$ in $\mathrm{K} 06$ can deviate significantly from the true one: interpretations of the observed proper motion measurements may or may not be correct. These results imply that the second interpretation is reasonable, though it remains still unclear how close the 3D space motions adopted in the tidal models are to the true ones.

Whether the first or the second interpretation is correct, a number of physical properties of the MS have not been clearly explained. In particular, the 'kink' in the LA around $b=0^{\circ}$ (C06) and the 'Magellanic filaments' (Nidever et al. 2007) have not been reproduced by any numerical models of the MS formation in a self-consistent manner. As suggested by Bekki et al. (2008), the origin of the kink around $b=0^{\circ}$ can result from the hydrodynamical interaction between the outer Hi disk of the Galaxy and the LA. The apparent physical connections between the Magellanic filaments and the LMC imply that there could be some interactions between the MS and the LMC. We plan to discuss these observations in Bekki \& Chiba (in preparation) based on more sophisticated models in which both a LMC-MS (or LMC-LA) hydrodynamical interaction and a Galaxy-LA one can be self-consistently investigated.

If the MS originates from the SMC, the gaseous abundances should be very similar to those of the outer gas disk of the SMC about 1.5 Gyr ago. The present chemodynamical models imply that the gaseous metallicity in $[\mathrm{Fe} / \mathrm{H}]$ ranges from -0.8 to -0.6 , which is significantly smaller than that of the $\mathrm{LMC}([\mathrm{Fe} / \mathrm{H}] \sim-0.3$, van den Bergh 2000). The details of abundance patterns (e.g. [C/Fe], $[\mathrm{N} / \mathrm{Fe}]$ and $[\mathrm{Mg} / \mathrm{Fe}])$ of the MS should be similar to those of the SMC about $1.5 \mathrm{Gyr}$ ago. We thus suggest that future extensive observational studies of abundance patterns of gas in the MS from QSO absorption lines will provide vital clues to the question as to whether the MS originate from the SMC or from the LMC. 